\title{
Noise Reduction for Modal Parameter Identification of the Measured FRFs Using the Modal Peak-Based Hankel-SVD Method
}

\author{
Tianxu Zhu, Chaoping Zang ${ }^{(D)}$, and Gengbei Zhang \\ College of Energy and Power Engineering, Nanjing University of Aeronautics and Astronautics, Nanjing 210016, China \\ Correspondence should be addressed to Chaoping Zang; c.zang@nuaa.edu.cn
}

Received 25 April 2020; Revised 5 June 2020; Accepted 8 July 2020; Published 25 July 2020

Academic Editor: Cristina Castejón

Copyright (C) 2020 Tianxu Zhu et al. This is an open access article distributed under the Creative Commons Attribution License, which permits unrestricted use, distribution, and reproduction in any medium, provided the original work is properly cited.

\begin{abstract}
The measured frequency response functions (FRFs) in the modal test are usually contaminated with noise that significantly affects the modal parameter identification. In this paper, a modal peak-based Hankel-SVD (MPHSVD) method is proposed to eliminate the noise contaminated in the measured FRFs in order to improve the accuracy of the identification of modal parameters. This method is divided into four steps. Firstly, the measured FRF signal is transferred to the impulse response function (IRF), and the Hankel-SVD method that works better in the time domain rather than in the frequency domain is further applied for the decomposition of component signals. Secondly, the iteration of the component signal accumulation is conducted to select the component signals that cover the concerned modal features, but some component signals of the residue noise may also be selected. Thirdly, another iteration considering the narrow frequency bands near the modal peak frequencies is conducted to further eliminate the residue noise and get the noise-reduced FRF signal. Finally, the modal identification method is conducted on the noise-reduced FRF to extract the modal parameters. A simulation of the FRF of a flat plate artificially contaminated with the random Gaussian noise and the random harmonic noise is implemented to verify the proposed method. Afterwards, a modal test of a flat plate under the high-temperature condition was undertaken using scanning laser Doppler vibrometry (SLDV). The noise reduction and modal parameter identification were exploited to the measured FRFs. Results show that the reconstructed FRFs retained all of the modal features we concerned about after the noise elimination, and the modal parameters are precisely identified. It demonstrates the superiority and effectiveness of the approach.
\end{abstract}

\section{Introduction}

In modal testing, the measured FRF signals are usually contaminated with noise $[1,2]$, and the noise will interfere with the accurate extraction of modal features. To eliminate the noise and purify the measured FRF signals, the noise reduction method should be exploited. In the past decades, many noise reduction methods such as the traditional low/ high-pass filter [3], singular value decomposition (SVD) $[4,5]$, minimum mean square error (MMSE) $[6,7]$, Wiener filter $[8,9]$, wavelet transform (WT) $[10,11]$, empirical mode decomposition (EMD) [12, 13], independent component analysis (ICA) [14, 15], and deep recurrent neural network (DRNN) [16, 17] have been developed. Among these methods, the SVD-related filters draw the most noticeable attention for their convenience, simplicity, and nonparametric properties and have been widely used in the noise reduction of various signals in engineering.
Generally speaking, the noised high-dimensional data, like the image signal [18] and multisensor signals [19], can be directly handled by the SVD filters without any preprocessing. But, difficulty exists for the low-dimensional data such as in the case of modal testing, in which only a few sensors are used to measure the frequency response functions (FRFs). In order to purify the measured FRF signals, the preprocessing techniques are conducted to improve their dimension before the SVD filter is subsequently carried out. The commonly used technique is the Hankel-SVD filter [20] that constructs a Hankel matrix from the separated single FRF signal to improve its dimension and then uses the SVD to purify the constructed Hankel matrix to reduce the noise of the correlated single FRF signal. The traditional HankelSVD filter mainly contains two steps [21]. The first step is the decomposition of the noise signal into the component signals and their correlated singular values using the HankelSVD method. The next is to select the useful component 
signals and the corresponding singular values to form the noise-reduced signal. In both steps, the decisive accuracy for the noise removal in the single FRF signal is how to select the useful singular values or the component signals.

In recent years, Yang and Tse [22] proposed a singular entropy selection method for singular value selection, which is to select the saturation point of the singular entropy calculated from the singular value sequences. Fan et al. [23] proposed a cluster method to select proper singular values, which is to select the big difference point of the standard deviation sequence calculated from the singular value clusters. Zhao and Ye [24] argued that the singular entropy is just like an inverse transform of the singular value sequence and proposed a difference spectrum of the singular value (DSSV) selection method to select the biggest point of the difference spectrum of the singular values. Bao et al. [25] introduced the model order indicator (MOI) to the singular value selection of the IRF signal for its noise reduction, which is to select the biggest point of the MOI spectrum calculated from singular values, and the formula of MOI is like an extension of DSSV. Although the above methods achieved good results in many cases, some drawbacks still exist. The above methods are based on the assumption that there will be a huge difference of the singular values produced by the noise and by the real signal. The big difference point between them can be used to select the number of singular values for denoising the signal. However, this assumption does not make any sense when dealing with the weak features in the signal because the big singular values do not represent the weak features sometimes and they do not contain a clear physical meaning.

Instead of using the biggest different point of the singular values to select the number of useful components, some new feature selection methods are based on the decomposed component signals from Hankel-SVD. Qiao and Pan [26] proposed a correlation coefficient selection method, which is to select the biggest difference point of the correlation coefficient spectrum calculated from the component signals and the original signal. The method shows a better result than the DSSV selection method. Zhao and Jia [27] proposed a periodic modulation intensity (PMI) method to select and enhance the fault feature of the rolling machine signal, which is to select the component signals whose impulse energy is bigger than noise energy for noise reduction. The result shows the method well enhances the impulse feature. Recently, Zhao and Ye [28] proved that the SVD has a feature to recover certain frequency components, and Cheng et al. [29] proposed a resonance enhancement SVD for feature extraction by adding the excitation sinusoidal signals with concerned frequencies to the original signal to form the enhanced signal. Then, a rectangle Hankel matrix with a certain dimension is constructed from the enhanced signal. The Hankel matrix is further decomposed into component signals using the SVD technique, and the first component signal, together with the excitation sinusoidal signals subtracted, is extracted to realize the feature extraction.

In this paper, a novel modal peak-based Hankel-SVD (MPHSVD) method is proposed to eliminate the noise and retain the whole concerned modal features of the FRF signals whose modal peak frequencies and modal peak amplitudes can be selected by observation. The MPHSVD is an iteration
Hankel-SVD filter, which contains the accumulation iteration and reselection iteration. The accumulation is to get the component signals of the transformed IRF signal that covers the whole concerned modal features, but these component signals will also cover some noise. The reselection is based on the accumulation result, which aims to separate the component signals which belong to the modal modes from the component signals of the noise and add them up to complete noise reduction thoroughly. Afterwards, the modal identification based on the rational fraction polynomial (RFP) method is conducted to the noise-reduced FRF signal to extract the modal parameters. The simulated and two experimental FRF signals of a flat plate with heavy noise are utilized to verify the approach. The results show the validity and superiority of the proposed method.

The rest of the paper is divided into four sections. In Section 2, the modal identification method based on the MPHSVD technique for noise reduction is represented. In Section 3, a simulated case is studied. In Section 4, the experimental cases are further used to verify the proposed method. The conclusion is drawn in the last section.

\section{Modal Parameter Identification of the Measured FRFs with Noise Reduction by MPHSVD}

In this section, the modal identification based on MPHSVD is described in detail, and the MPHSVD method is the Hankel-SVD filter with the modal peak-based component signal selection method, which is used for eliminating the noise of the single FRF signal.

2.1. Hankel-SVD of the Transformed IRF Signal. As the transformed impulse response function (IRF) signal is better in the noise reduction than the FRF signal, the FRF signal $H(\omega)$ is therefore transformed to the IRF signal by inverse Fourier transform:

$$
h(t)=\operatorname{iFT}(H(\omega))
$$

where $h(t)$ represents the transformed IRF signal and iFT represents the inverse Fourier transform. Besides, the inverse Fourier transform is conducted in the fast Fourier transform framework, so the transformed IRF signal correlated with the $N / 2$ length long FRF signal $H(\omega)$ contains $N$ points.

After the transformed IRF signal is obtained, the HankelSVD is conducted to decompose the IRF signal into component signals. For the length $N$ transformed IRF signal $h(t)$,

$$
h(t)=\left[\begin{array}{llll}
h_{1} & h_{2} & \cdots & h_{N}
\end{array}\right]
$$

its Hankel matrix $A$ is

$$
A=\left[\begin{array}{cccc}
h_{1} & h_{2} & \cdots & h_{n} \\
h_{2} & h_{3} & \cdots & h_{n+1} \\
\vdots & \vdots & \ddots & \vdots \\
h_{m} & h_{m+1} & \cdots & h_{N}
\end{array}\right],
$$


where $m$ is the row number of the Hankel matrix $A, n$ is the column number, and $m+n-1=N$. Here, $m$ is set as floor (N/2) to make the Hankel matrix nearly square, where the symbol "floor" means rounding down to the nearest integer. The reason of setting the Hankel matrix nearly square is that a nearly square Hankel matrix can achieve the maximum noise reduction quality of the Hankel-SVD filter. Then, matrix $A$ can be decomposed by the SVD technique:

$$
A=U_{m \times m} S_{m \times n} V_{n \times n}^{T}=\left[\begin{array}{llll}
u_{1} & u_{2} & \cdots & u_{m}
\end{array}\right]\left[\begin{array}{cccc}
\sigma_{1} & 0 & \cdots & 0 \\
0 & \sigma_{2} & \cdots & 0 \\
\vdots & \vdots & \ddots & \vdots \\
0 & 0 & \cdots & \sigma_{q} \\
0 & 0 & 0 & 0
\end{array}\right]\left[\begin{array}{c}
v_{1}^{T} \\
v_{2}^{T} \\
\vdots \\
v_{n}^{T}
\end{array}\right] \text {, }
$$

where $U$ is the left orthogonal matrix, $u_{i}$ is the column vector of $U, V$ is the right orthogonal matrix, $v_{i}$ is the column vector of $V, S$ is the singular value matrix, and $\sigma_{i}$ is the $i^{\text {th }}$ singular value. The dimension of $S$ is $q$, and $q$ is equal to $\min (m, n)$. Then, by expanding equation (4), we get the following equation:

$$
A=\sigma_{1} u_{1} v_{1}^{T}+\sigma_{2} u_{2} v_{2}^{T}+\cdots+\sigma_{q} u_{q} v_{q}^{T}=A_{1}+A_{2}+\cdots+A_{q},
$$

where $A$ is decomposed to the summation of $q$ component matrices and $A_{i}$ is the $i^{\text {th }}$ component matrix of the component matrix set. The dimension of $A_{i}$ is equal to the dimension of $A$, which is $m \times n$. Besides, we assume the component matrix still contain the Hankel structure, and matrix $A_{i}$ is shown in the following equation:

$$
A_{i}=\left[\begin{array}{cccc}
s_{1,1}^{i} & s_{1,2}^{i} & \cdots & s_{1, n}^{i} \\
s_{2,1}^{i} & s_{2,2}^{i} & \cdots & s_{2, n}^{i} \\
\vdots & \vdots & \ddots & \vdots \\
s_{m, 1}^{i} & s_{m, 2}^{i} & \cdots & s_{m, n}^{i}
\end{array}\right]=\left[\begin{array}{cccc}
h_{1}^{i} & h_{2}^{i} & \cdots & h_{n}^{i} \\
h_{2}^{i} & h_{3}^{i} & \cdots & h_{n+1}^{i} \\
\vdots & \vdots & \ddots & \vdots \\
h_{m}^{i} & h_{m+1}^{i} & \cdots & h_{N}^{i}
\end{array}\right],
$$

where $s_{a, b}^{i}$ represents the element of the $a^{\text {th }}$ row and the $b^{\text {th }}$ column in matrix $A_{i}$ and $A_{i}$ contains $m n$ elements. Due to the Hankel structure of $A_{i}$, the values of the elements in the antidiagonal line are actually the same, so the elements in each antidiagonal line can be represented by one element. Thus, the whole $m n$ elements in $A_{i}$ can be represented by $N$ elements because there are totally $N$ antidiagonal lines. Besides, $N$ is equal to $m+n-1$, and $h_{j}^{i}$ represents the element of the $j^{\text {th }}$ antidiagonal line of matrix $A_{i}$. Then, we define the first row of $A_{i}$ as $L_{i}$ and define the last column without the first element of $A_{i}$ as $R_{i}$. The elements of $L_{i}$ and $R_{i}$ can form a component signal $h^{i}(t)$, which is correlated with its singular value $\sigma_{i}$ :

$$
\begin{aligned}
& L_{i}=\left[\begin{array}{cccc}
h_{1}^{i} & h_{2}^{i} & \cdots & h_{n}^{i} \\
\hline A_{i}^{i} & h_{3}^{i} & \cdots & h_{n+1}^{i} \\
\vdots & \vdots & \ddots & \vdots \\
h_{m}^{i} & h_{m+1}^{i} & \cdots & h_{N}^{i}
\end{array}\right]=R_{i}, \\
& h^{i}(t)=\left[\mathrm{L}_{i}, R_{i}^{T}\right]=\left[h_{1}^{i} \cdots h_{n}^{i} h_{n+1}^{i} \cdots h_{N}^{i}\right] .
\end{aligned}
$$

Then, because Hankel matrix $A$ can be divided into the summation of the component matrices, $A=A_{1}+A_{2}+\cdots+A_{q}$, the first row $L$ and the final column $R$ of matrix $A$ can also be divided to the sum of the first rows and the last columns of the component matrices according to the properties of matrix addition:

$$
\begin{aligned}
& L=\left[\begin{array}{llll}
h_{1} & h_{2} & \cdots & h_{n}
\end{array}\right]=\sum_{i=1}^{q} L_{i}=\left[\begin{array}{cccc}
h_{1}^{1} & h_{2}^{1} & \cdots & h_{n}^{1} \\
+ & + & + & + \\
\vdots & \vdots & \vdots & \vdots \\
+ & + & + & + \\
h_{1}^{q} & h_{2}^{q} & \cdots & h_{n}^{q}
\end{array}\right], \\
& R=\left[\begin{array}{llll}
h_{n+1} & h_{n+2} & \cdots & h_{N}
\end{array}\right]=\sum_{i=1}^{q} R_{i}=\left[\begin{array}{cccc}
h_{n+1}^{1} & h_{n+2}^{1} & \cdots & h_{N}^{1} \\
+ & + & + & + \\
\vdots & \vdots & \vdots & \vdots \\
+ & + & + & + \\
h_{n+1}^{q} & h_{n+2}^{q} & \cdots & h_{N}^{q}
\end{array}\right]^{T}
\end{aligned}
$$

Finally, the IRF signal $h$ is decomposed to the component signals formed by $L_{i}$ and $R_{i}$ because the signal $h$ can be represented by the combination of $L$ and $R$ :

$$
\begin{aligned}
h(t) & =[L, R]=\left[\sum_{i=1}^{q} L_{i}, \sum_{i=1}^{q} R_{i}\right]=\left[\begin{array}{cccccc}
h_{1}^{1} & h_{2}^{1} & \cdots & h_{n}^{1} & \cdots & h_{N}^{1} \\
+ & + & \cdots & + & \cdots & + \\
\vdots & \vdots & \vdots & \vdots & \vdots & \vdots \\
+ & + & \cdots & + & \cdots & + \\
h_{1}^{q} & h_{2}^{q} & \cdots & h_{n}^{q} & \cdots & h_{N}^{q}
\end{array}\right] \\
& =\sum_{i=1}^{q} h^{i}(t) .
\end{aligned}
$$

The above derivation is based on the assumption that component matrices still contain the Hankel structure after SVD, but the decomposition result of SVD does not actually follow the assumption. Thus, we usually average the antidiagonal lines of the component matrices such that they contain the Hankel structure and get the component signals. The antidiagonal averaging of the component matrix $A_{i}$ is shown in the following equation:

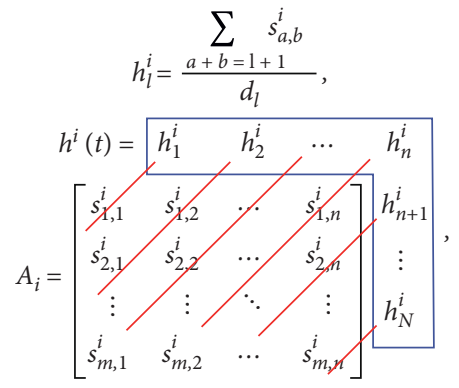

where the averaging of the $t^{\text {th }}$ antidiagonal line means adding up the elements whose coordinate summation in $A_{i}$ is equal to $l+1$ and dividing the total element number $d_{l}$ to get the element $h_{l}^{i}$. The red lines in the equation mean averaging, 
and the equation means averaging each antidiagonal line of $A_{i}$ to get the elements of $h^{i}$.

\subsection{Selection of the Number of the Accumulated Component} Signals. Based on the Hankel-SVD technique, the frequencies and the amplitudes at modal peaks of the FRF signal can be decomposed to the component signals [28]. The component signals are arranged in orders corresponding to the singular values sorted from the largest to the smallest. Generally, one modal frequency mode can be decomposed into one or several pairs of adjacent component signals, and one pair of adjacent component signals correlated with the largest pair of singular values of this mode is likely dominated.

For the experimental FRF signal $H(\omega)$, the concerned number of modal peaks is set as $k$. Then, the concerned modal peak frequencies, $f=\left[\begin{array}{llll}f_{1} & f_{2} & \cdots & f_{k}\end{array}\right]$, and their amplitudes, $R=\left[\begin{array}{llll}R_{1} & R_{2} & \cdots & R_{k}\end{array}\right]$, can be selected. Considering all the decomposed component signals of the IRF signal, the iteration of the component signal accumulation is conducted. For the $i^{\text {th }}$ iteration, the $i^{\text {th }}$ component signal $h^{i}$ is added to the $(i-1)^{\text {th }}$ accumulated signal $h_{\text {acu }}^{i-1}$ to form the $i^{\text {th }}$ accumulated signal $h_{\text {acu }}^{i}$ :

$$
h_{\mathrm{acu}}^{i}=h_{\mathrm{acu}}^{i-1}+h^{i} .
$$

Afterwards, the energy spectrum of the accumulated signal $h_{\mathrm{acu}}^{i}$ is calculated by Fourier transform:

$$
E_{i}=\mathrm{FT}\left(h_{\mathrm{acu}}^{i}\right)
$$

where $E_{i}$ represents the energy spectrum of the accumulated signal $h_{\mathrm{acu}}^{i}$.

Then, the differences between modal peak amplitudes $R$ and the amplitudes of energy spectrum $E_{i}$ at modal peak frequencies $f$ are calculated:

$$
D_{i}=\operatorname{abs}\left(E_{i}(f)-R\right) \cdot / R=\left[\begin{array}{llll}
D_{1}^{i} & D_{2}^{i} & \cdots & D_{k}^{i}
\end{array}\right],
$$

where the symbol "abs" represents the absolute value, the symbol "./" means the vector point division, $D_{i}$ is the $i^{\text {th }}$ difference vector, and $D_{l}^{i}$ is the difference between the $l^{\text {th }}$ modal peak amplitude $R_{l}$ and the energy spectrum $E_{i}$ at the modal peak frequency $f_{l}$.

Finally, $D_{i}$ is judged by the threshold $T$. If the arbitrary component of vector $D$ of the $i^{\text {th }}$ iteration is below or equal to the threshold $T$,

$$
\left[\begin{array}{c}
D_{1}^{i} \leq T \\
D_{2}^{i} \leq T \\
\vdots \\
D_{k}^{i} \leq T
\end{array}\right],
$$

the iteration stops, and the rest of the component signals are treated as noise. If not, the next iteration will be conducted.

After the accumulation ends, the whole modal modes will recover, and the number of the selected component signals is recorded as $m$. However, some residue noise may also be recovered in the component signal accumulation. To eliminate the residue noise, and accomplish the noise elimination thoroughly, a further reselection method will be conducted.
2.3. Component Signal Reselection for Eliminating the Residue Noise. The peak frequencies of the component signals correlated with the modes are near the modal peak frequencies, while the peak frequencies of the component signals correlated with the residue noise will be away from the modal peak frequencies in some degrees. Thus, if some narrow frequency bands are set near the peak frequencies, the residue noises can be eliminated by reselecting the component signals whose peak frequencies are in the bands from the accumulation result for noise reduction.

For there are $k$ cared modal frequencies $\left[f_{1}, f_{2}, \ldots, f_{k}\right]$ in $H$, and $k$ frequency bands with the bandwidth $d$ are set as the passband $P$ :

$$
P=\left[\begin{array}{c}
F_{1} \\
F_{2} \\
\vdots \\
F_{k}
\end{array}\right]=\left[\begin{array}{c}
\operatorname{band}\left(f_{1}-\frac{d}{2}, f_{1}+\frac{d}{2}\right) \\
\operatorname{band}\left(f_{2}-\frac{d}{2}, f_{2}+\frac{d}{2}\right) \\
\vdots \\
\operatorname{band}\left(f_{1}-\frac{d}{2}, f_{1}+\frac{d}{2}\right)
\end{array}\right] .
$$

Since there are $m$ component signals extracted from the component signal accumulation, another iteration with the max iteration number $m$ is conducted to reduce the residual noise, and the $j^{\text {th }}$ iteration is shown below.

Firstly, for the $j^{\text {th }}$ component signal $h^{j}$ extracted from the accumulation, its correlated energy spectrum is calculated by the Fourier transform, which is shown in the following equation:

$$
E_{c}^{j}=\mathrm{FT}\left(h^{j}\right)
$$

where $E_{c}^{j}$ is the energy spectrum of the $j^{\text {th }}$ component signal $h^{j}$ and FT represents the Fourier transform. Then, the frequency of the peak amplitude in the energy spectrum $E_{c}^{j}$ is extracted:

$$
f_{\max }^{j}=\max \left(E_{c}^{j}\right) \text {, }
$$

where $f_{\max }^{j}$ is the frequency of the max amplitude of $E_{c}^{j}$. If the frequency $f_{\max }^{j}$ is in the passband $P$, the component signal is added to the second accumulated signal:

$$
h_{\mathrm{acu} 2}=h_{\mathrm{acu} 2}+h^{j} \text {. }
$$

If $f_{\max }^{j}$ is not in the band, the component signal $h^{j}$ is abandoned, and the next iteration is conducted. When the iteration turn is $m$, the iteration ends, and the second accumulated signal $h_{\text {acu2 }}$ is signed as the noise-reduced IRF signal $\widetilde{h}$. The noise-reduced FRF $\widetilde{H}$ is transformed from it:

$$
\begin{aligned}
\tilde{h} & =h_{\text {acu2}}, \\
\widetilde{H} & =\operatorname{FT}(\widetilde{h}) .
\end{aligned}
$$

2.4. Modal Parameter Identification with the Noise-Reduced FRF. After the noise reduction, the noise-reduced FRF 
signal $\widetilde{H}$ is acquired, and the modal parameters are subsequently extracted by the modal identification. The rational fraction polynomial (RFP) method that is widely used in the modal test package is selected here. One benefit of using the RFP method is that it is suitable for a single FRF to extract modal parameters. Since the method is well developed, and the main formulas of the method are described thoroughly in reference [30], more detailed description of the method is not given here.

In summary, the framework of the modal identification by MPHSVD is given in Figure 1. It is divided into four steps as follows:

Step 1: transform the noised FRF signal to noised IRF signal by IFFT and decompose it to $n$ component signals and the corresponding $n$ singular values by Hankel-SVD

Step 2: accumulate the component signals in the order of the corresponding singular values from the largest to the smallest to get the first $m$ component signals that cover the modal modes and some residue noise

Step 3: reselect $q$ component signals of the modal modes from the first $m$ component signals and add them up to get the noise-reduced IRF signal and the corresponding FRF signal

Step 4: identify the modal parameters from the noisereduced FRF signal by modal identification

\section{Case Study I: Simulation}

3.1. The Simulated FRF Signal with the Added Noise. To verify the proposed method, a simulation of a steel flat plate is carried out using the finite element (FE) analysis. An FRF signal of one point at the middle left of the plate is simulated. The calculated frequency band is $[0 \sim 3200] \mathrm{Hz}$ with an interval of $0.5 \mathrm{~Hz}$. Therefore, the FRF signal contains 6400 points with a maximum frequency of $3200 \mathrm{~Hz}$. The corresponding impulse response function (IRF) is attained by the inverse Fourier transform from the FRF signal and contains 12800 points. The simulated FRF signal and its corresponding IRF signal are shown in Figure 2.

In Figure 2(a), we can clearly see seven frequencies at the modal peaks which are $64 \mathrm{~Hz}, 399 \mathrm{~Hz}, 428 \mathrm{~Hz}$, $1119 \mathrm{~Hz}, 1334 \mathrm{~Hz}, 2195 \mathrm{~Hz}$, and $2381 \mathrm{~Hz}$, respectively. Figure 2(b) is the transformed IRF signal. It can be seen that the amplitude of the transformed IRF signal does not ideally decrease to zero since the IRF signal is taken from the real part of the discrete inverse Fourier transform of the simulated FRF signal. This will cause some digital errors and make the energy spectrum of the transformed IRF signal slightly different from the energy spectrum of the original FRF signal. Besides, the proposed method is mainly conducted on the transformed IRF signal, so the frequencies and the amplitudes at the modal peaks mentioned in this paper are selected from the energy spectrum of the transformed IRF signal instead of the energy spectrum of the original FRF signal for accuracy. Then, to simulate the IRF signal contaminated with noise, the additive noise is added to the IRF signal by the following equation:

$$
h_{\text {noise }}(t)=h(t)+A_{n} \times n(t)+\sum_{i=1}^{35} A_{n i} \cos \left(2 \pi f_{n i} t\right),
$$

where $h_{\text {noise }}(t)$ is the noised IRF signal, $h(t)$ is the noise-free IRF signal, $n(t)$ is the random Gaussian noise, $A_{n}$ is the amplitude of the random noise, which is set as two point five percent of the max amplitude of the noise IRF signal $h(t)$, and $A_{n i} \cos \left(2 \pi f_{n i} t\right)$ is the random harmonic noise; its frequency is obtained from the frequency band $[100,400]$, $[500,1000],[1500,2000]$, and $[2500,3000]$ randomly. Its amplitude is randomly obtained from the amplitude band $\left[0,0.05 A_{\max }\right]$, where $A_{\max }$ is the max amplitude of the noisefree IRF signal $h(t)$. After the noise signals are added to the noise-free IRF signal, the SNR between the original IRF signal and the added noise is 0.4691 , and the noise IRF signal with its correlated noised FRF signal is shown in Figure 3.

Obviously, the FRF signal in Figure 3(a) is polluted heavily by the noise. The energy spectrum of the noised FRF signal severely distorts from the noise-free one, and the phase spectrum is totally drown into the noise, where many of the phase changes are hard to be seen directly. From Figure 3(b), it can be seen that the noise pollutes most parts of the IRF signal, which makes the noised IRF signal deeply distort from the simulated IRF signal. Thus, the qualities of the FRF and IRF signals are seriously damaged by the noise. Then, the real modal parameters are extracted from the original noise-free FRF signal using the RFP method, and the noised modal parameters are extracted from the noised FRF signal using the same curve fitting frequency intervals of the noise-free FRF signal. The curve fittings are plotted in Figure 4, and the identified modal parameters are listed in Table 1. Figures 4(a) and 4(b) show the curve fitting of the original FRF signal, and the fitted curve matches the original FRF signal well. The curve fitting of the noised FRF signal is shown in Figures 4(c) and 4(d). It can be found that there are some differences in the modal peak frequencies 1 and 5 between the fitted curve and the noised FRF in the energy spectrum. Besides, by comparing the fitted curve and the noise-free FRF signal, there is some difference between the fitted curve and the noise-free FRF near modes 2 and 3. Thus, the fitted curve is inaccurate. From Table 1, the modal parameters of the noised FRF and real modal parameters extracted from the original noise-free FRF signal are given. Besides, the differences between them are also calculated. The frequency differences are the absolute differences between the modal frequencies of the two FRF signals. The damping factor differences are calculated by the following equation:

$$
D_{i}^{\mathrm{damp}}=\frac{\operatorname{abs}\left(\xi_{i}^{\text {noisefree }}-\xi_{i}^{\text {noised }}\right)}{\xi_{i}^{\text {noisefree }}},
$$

where $D_{i}^{\text {damp }}$ is the damping factor difference of the $i^{\text {th }}$ mode, "abs" means the absolute value, $\xi_{i}^{\text {noisefree }}$ is the damping factor from the noise-free FRF signal, and $\xi_{i}^{\text {noised }}$ is the damping factor of the noised FRF signal. Similarly, there is 


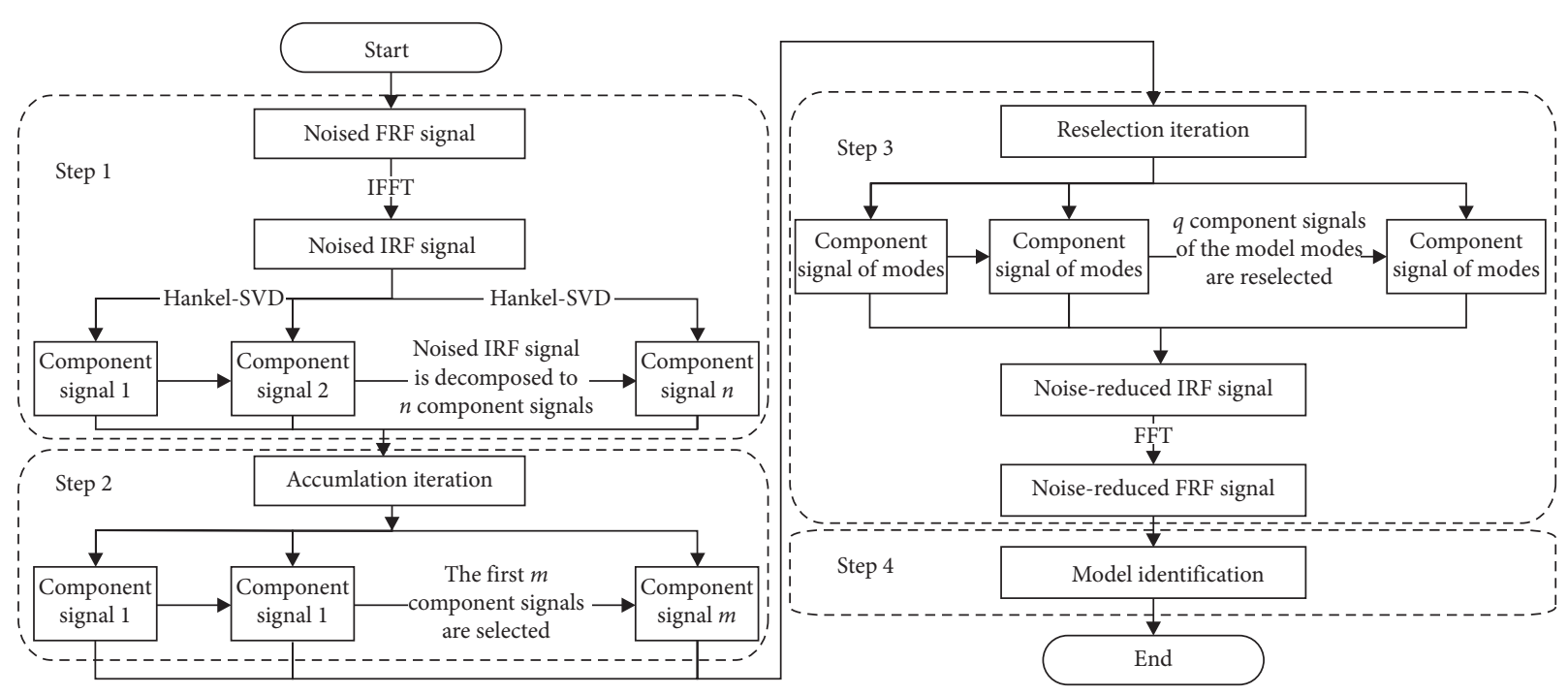

Figure 1: The flowchart of the proposed method.

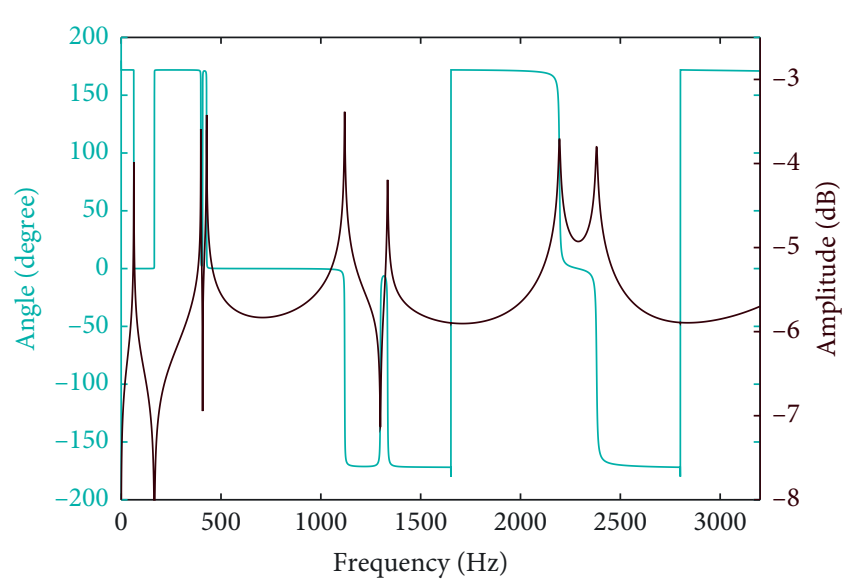

(a)

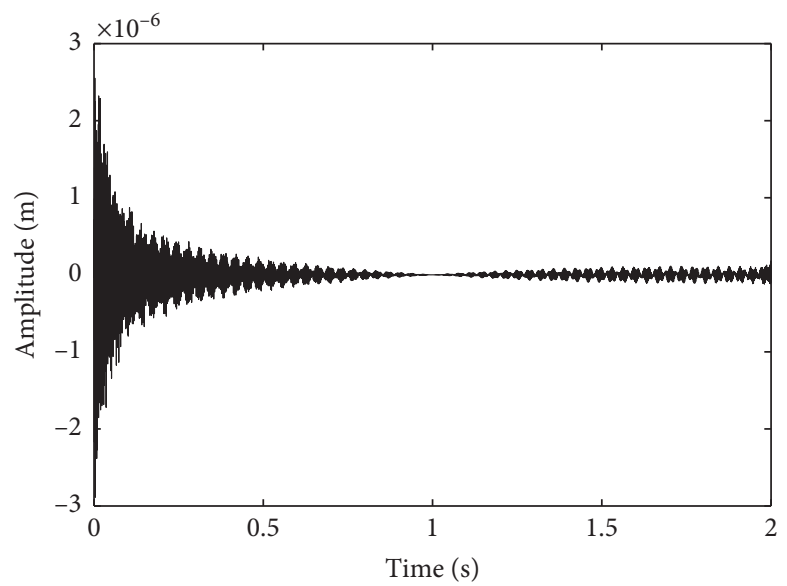

(b)

Figure 2: (a) Simulated FRF signal. (b) Simulated IRF signal.

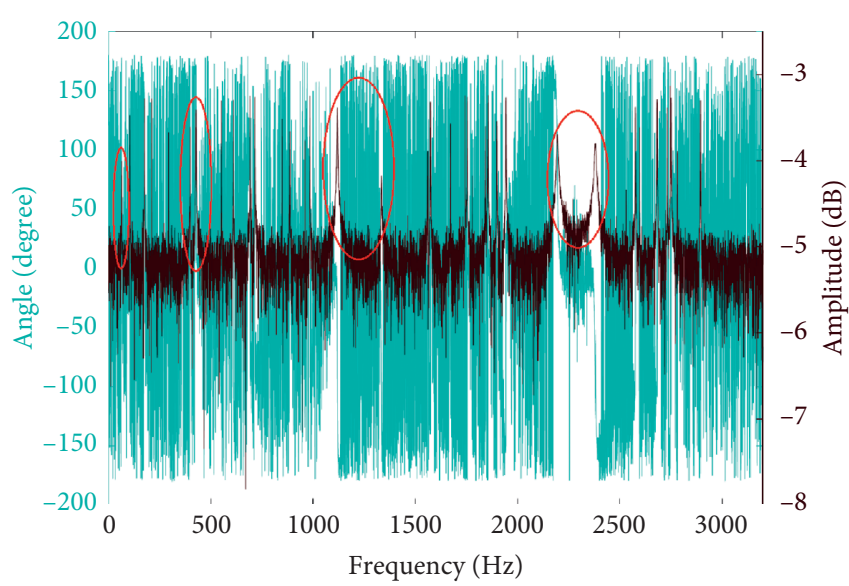

(a)

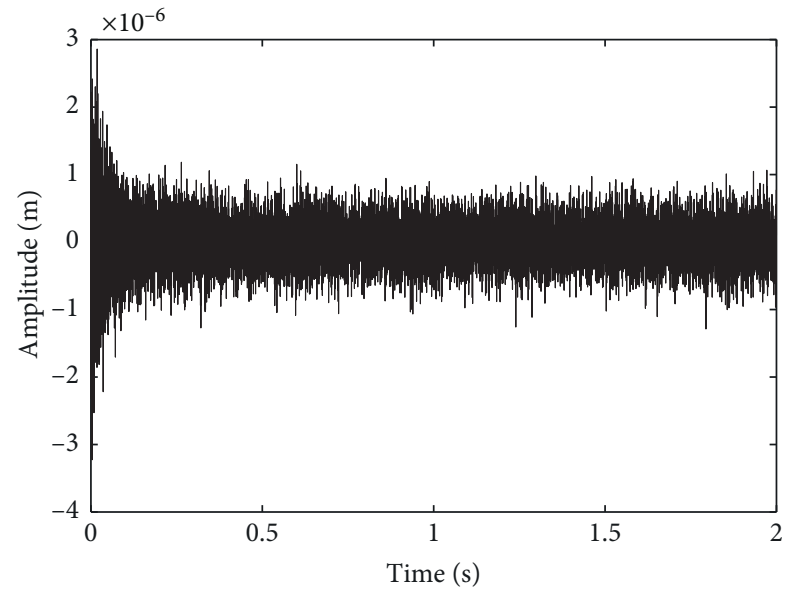

(b)

FIGURE 3: (a) FRF signal with the added noise. (b) IRF signal with the added noise. 


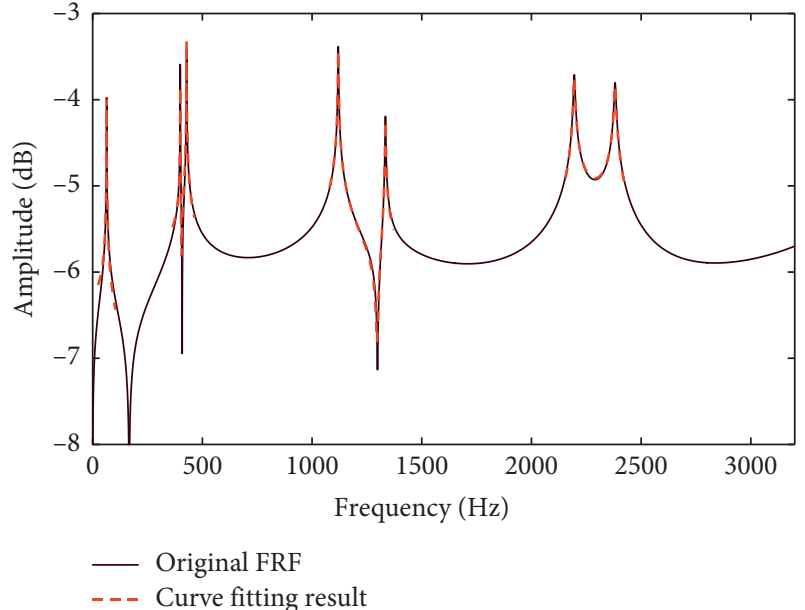

(a)

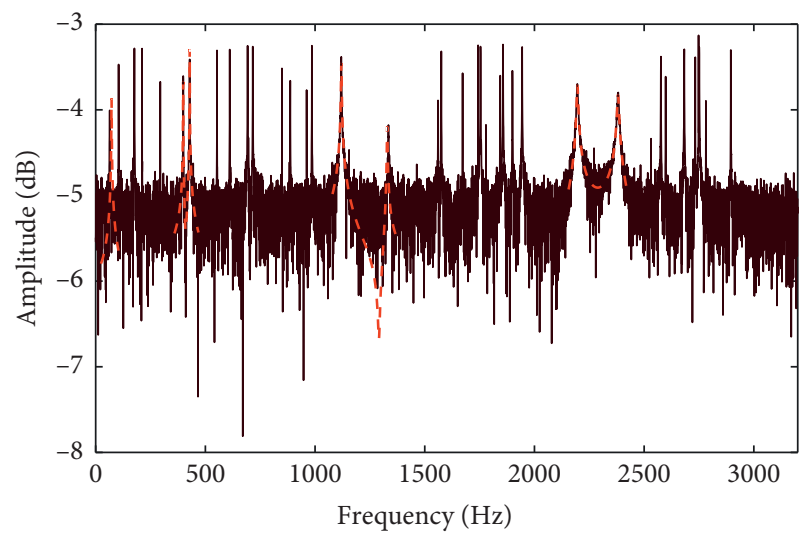

$\begin{array}{ll}\text { - Noised FRF } \\ \text { - } & \text { Curve fitting result }\end{array}$

(c)

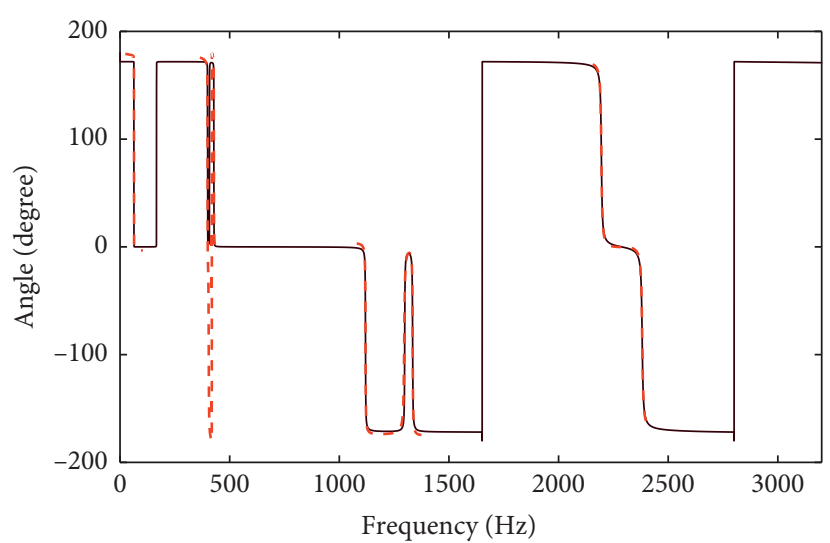

(b)

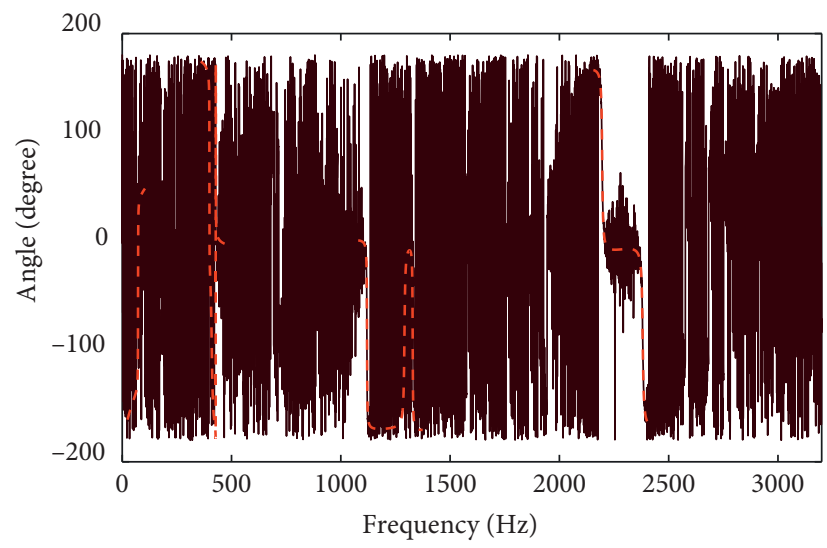

(d)

FIGURE 4: Curve fitting results of the noised and noise-free FRF signals. (a) Curve fitted energy spectrum of original FRF. (b) Curve fitted phase spectrum of original FRF. (c) Curve fitted energy spectrum of noised FRF (d) Curve fitted phase spectrum of noised FRF.

TABLE 1: Modal parameters of the noised FRF signal.

\begin{tabular}{lcccccc}
\hline Mode & $\begin{array}{c}\text { Modal frequency } \\
(\mathrm{Hz})(\text { original })\end{array}$ & $\begin{array}{c}\text { Modal frequency } \\
(\mathrm{Hz})(\text { noised })\end{array}$ & $\begin{array}{c}\text { Frequency difference } \\
(\mathrm{Hz})\end{array}$ & $\begin{array}{c}\text { Damping factor } \\
\text { (original) }\end{array}$ & $\begin{array}{c}\text { Damping factor } \\
(\text { noised })\end{array}$ & $\begin{array}{c}\text { Damping factor } \\
\text { difference }(\%)\end{array}$ \\
\hline 1 & 63.67794 & 72.34572 & 8.66778 & 0.000674 & -0.00682 & 1111.957 \\
2 & 399.3322 & 398.8602 & 0.472005 & 0.000295 & 0.000769 & 160.5308 \\
3 & 428.0756 & 428.581 & 0.505418 & 0.000667 & 0.000515 & 22.80982 \\
4 & 1119.442 & 1119.783 & 0.340427 & 0.000866 & 0.000991 & 14.50155 \\
5 & 1334.573 & 1328.813 & 5.760097 & 0.000943 & 0.000941 & 0.250468 \\
6 & 2195.12 & 2195.789 & 0.66933 & 0.001474 & 0.00135 & 8.443071 \\
7 & 2381.407 & 2381.004 & 0.402907 & 0.001588 & 0.001627 & 2.482244 \\
\hline
\end{tabular}

nearly a $8 \mathrm{~Hz}$ difference in mode 1 between the extracted modal frequency and the real modal frequency. Besides, there is a $6 \mathrm{~Hz}$ difference in mode 5 between the extracted and the real modal frequency. The extracted damping factor of mode 1 is negative, and the difference between damping factors of mode 1 extracted from the noise-free and noised FRFs is 11 times bigger than the real damping factor of mode 1 . It is noticed that the damping factor difference of mode 1 is far beyond the damping factor differences of the other modes. It is because that the mode 1 has the lowest energy of all concerned modes, and it is heavily affected by the noise. Besides, due to the inaccurate curve fitting of mode 2, the damping factor extracted from the noised FRF signal is nearly 2.6 times bigger than the factor of the noise-free FRF signal. Then, the analysis of the noised FRF signal shows that the quality of the FRF signal is 
damaged by the noise seriously, and the extracted modal parameters are inaccurate.

\subsection{The Noise Reduction of the Simulated FRF Signal Using the} MPHSVD Method. To eliminate the noise contaminated in the FRF signal, the corresponding noised IRF signal in Figure $3(\mathrm{~b})$ is taken into consideration. Firstly, a nearly square Hankel matrix with 6400 rows and 6401 columns is constructed from the noised IRF signal. The Hankel matrix is subsequently decomposed into 6400 component signals using the SVD technique. The seven modal frequencies and their amplitudes are selected for noise elimination. The amplitude difference threshold $T$ is set as 0.15 , and the iteration of the component signal accumulation is conducted to recover the selected modal peak amplitudes and modal peak frequencies. The iteration stops at the $80^{\text {th }}$ iteration turn, and the first 80 component signals are accumulated to form the accumulated signal. Then, the accumulated signal is transformed to the frequency domain to acquire the correlated FRF signal, and the overlay between the FRF signal from the accumulated signal and the FRF signal from the transformed noise-free IRF signal is given in Figure 5. Obviously, seven modal frequencies and their amplitudes are recovered from Figure 5(a), which is marked in red circles in the figure. Clearly, the random Gaussian noise is mostly eliminated, but many random harmonic noises are simultaneously recovered after the accumulation, which makes it hard to distinguish which peaks are correlated with the real modal modes. The phase spectrum after accumulation is shown in Figure 5(b). Similarly, the phase changes correlated with the modal modes are recovered, and the changes of the harmonic noises are also recovered, which makes the phase spectrum still distort from the noise-free one a lot. Then, from Figure 5, the accumulation removes most parts of the random Gaussian noise, but it recovers most parts of the random harmonic noises with the recovery of the modal modes. To eliminate the residue harmonic noises and complete the noise reduction thoroughly, the further component signal reselection is conducted.

Because seven selected modal peaks are supposed to be kept after noise recovery, seven frequency bands with the bandwidth $35 \mathrm{~Hz}$ near the seven modal peak frequencies are set as the threshold for the further noise removal. The component signals, that the frequency of the biggest peak amplitude is in the set frequency bands, are reselected for the further noise reduction. Then, for the 80 component signals selected from accumulation, the component signal reselection is conducted for them to separate the component signals correlated with the modal modes for recovery and abandon the component signals of the random harmonic noises. Finally, 20 component signals are reselected, and they are added up to form the noise-reduced IRF signal. The overlays of the noise-reduced IRF signal against the noised IRF signal and the noise-reduced IRF signal against the noise-free IRF signal are given in Figures 6(a) and $6(b)$.
It can be found that most parts of the noise are removed, and there is mostly no difference between the noise-reduced and the noise-free IRF signals, except the part of the red circle in the figure. Then, a noise removal rate is calculated by the following equation

$$
\mathrm{NR}=1-\frac{\operatorname{sum}\left(\operatorname{abs}\left(h_{\text {denoised }}-h\right)\right)}{\operatorname{sum}(\operatorname{abs}(h))},
$$

where $h_{\text {denoised }}$ is the noise-reduced IRF signal and NR is the noise removal rate. The calculation result from Figure 6(b) is 0.9365 , which indicates that $93.65 \%$ of the noise is removed. Then, the noise-reduced IRF signal is transformed to the noise-reduced FRF signal, and the comparison between the noise-reduced FRF signal and the FRF signal from the noisefree IRF signal is shown in Figure 7. The noise-reduced energy spectrum comparison is shown in Figure 7(a). It is obvious that the random harmonic noises are eliminated, where the seven modal peaks are the only peaks that can be seen directly. Besides, the noise-reduced spectrum fits the noise-free one well, which shows the validity of the noise elimination. Figure $7(\mathrm{~b})$ gives the noise-reduced phase spectrum of the reselected 22 component signals, the phase changes correlated with the modal features are recovered, and they fit the noise-free one well. From the analysis of the noise reduction result, the MPHSVD filter successively eliminates the random Gaussian noise and the random harmonic noises thoroughly. Besides, the final noise reduction result fits the noise-free one well, which shows the validity of the proposed method.

3.3. Detailed Analysis of the Noise Reduction Method. For more detailed analysis of the relationship between the modal modes, the harmonic noises, and the combination of component signals, a part of the first 80 component signals are investigated, and the result is shown in Figure 8. The combinations of reselected 20 component signals are correlated with the modal modes, which are given in Figures $8(\mathrm{a})$ to $8(\mathrm{~g})$, and the other 60 component signals are correlated with the noises, where the combinations of component signals correlated with two harmonic noises are shown in Figures $8(\mathrm{~h})$ and $8(\mathrm{i})$. Figures $8(\mathrm{a})$ and $8(\mathrm{~b})$ give the energy spectra of the component signals which form the $1^{\text {st }}$ and $2^{\text {nd }}$ modes. It can be found that the first mode at $65 \mathrm{~Hz}$ is decomposed into two adjacent component signal pairs of component signals $75,76,77$, and 78 . The pair of component signals 75 and 76 is dominant. Mode 2 is decomposed into two component signal pairs, and the dominant pair of component signals 55 and 56 takes $88 \%$ of the energy of the mode. Figures $8(\mathrm{c})$ and $8(\mathrm{~d})$ give the energy spectra correlated with the $3^{\text {rd }}$ and $4^{\text {th }}$ modes. The $3^{\text {rd }}$ mode is decomposed into a dominant pair of component signals 41 and 42 and a nondominant pair of component signals 63 and 64. Mode 4 is decomposed into the pair of component signals 37 and 38 . Figures $8(\mathrm{e}), 8(\mathrm{f})$, and $8(\mathrm{~g})$ give the energy spectra of the component signals for modes 5,6 , and 7. Each of the three modes is decomposed into one pair of component signals. Mode 5 is decomposed into the component signals 79 and 80 . Mode 6 is decomposed into the pair of 


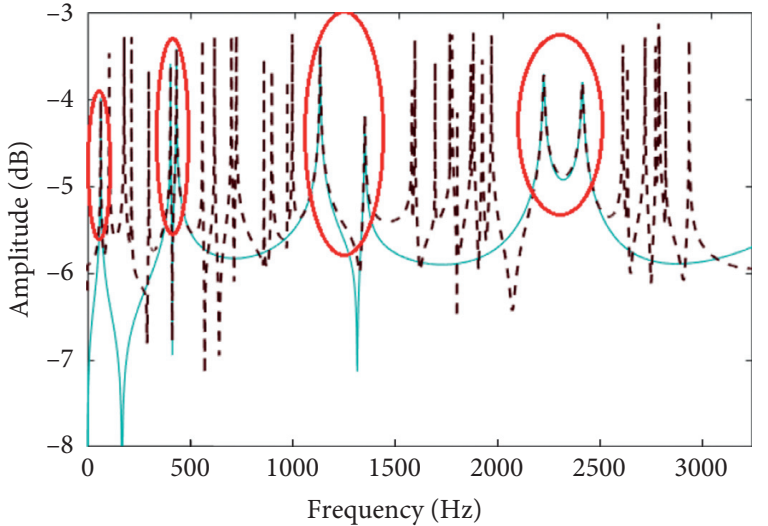

FRF signal from the noise-free IRF signal

- - - FRF signal from the accumulated signal

(a)

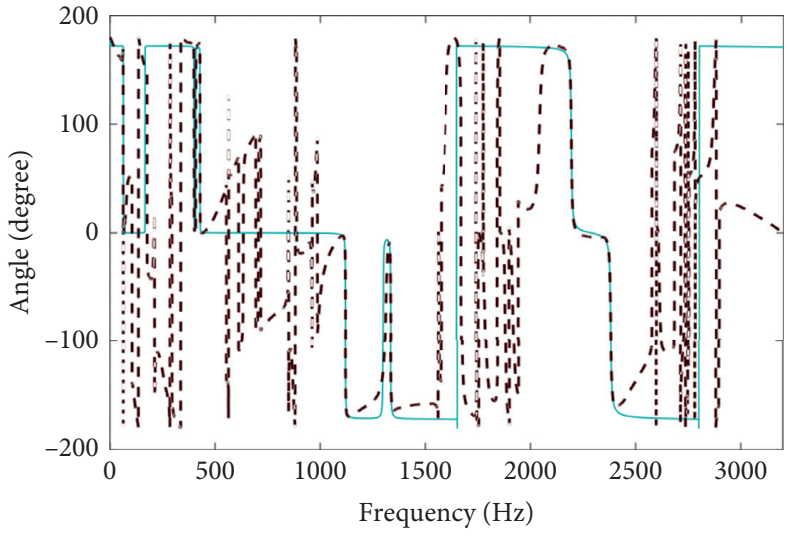

(b)

Figure 5: Noise reduction result of the component signal accumulation. (a) Energy spectrum. (b) Phase spectrum.

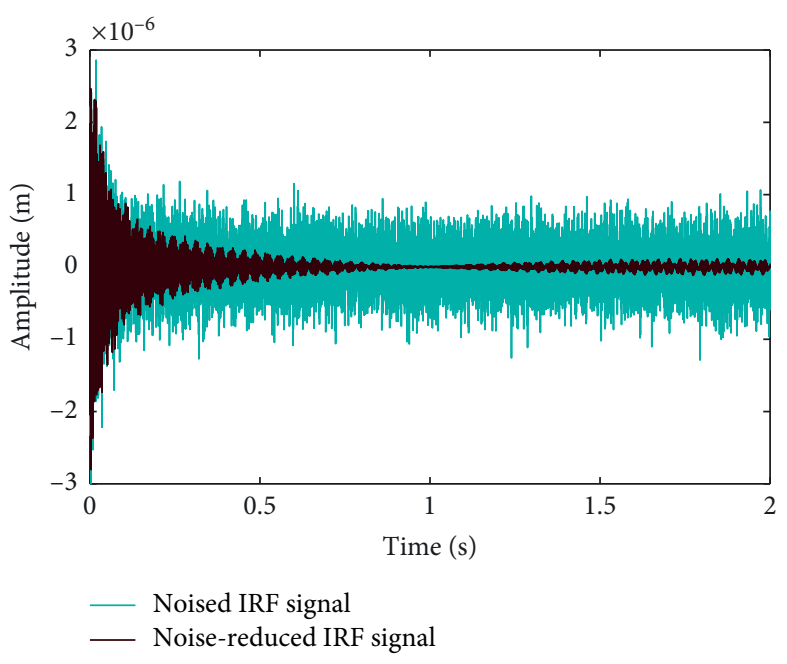

(a)

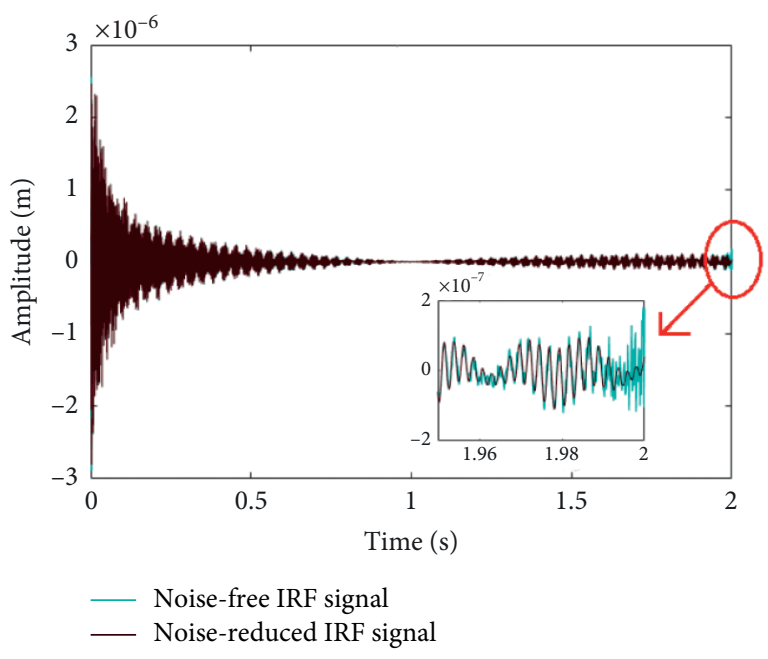

(b)

FIGURE 6: (a) Overlay of the noised IRF signal. (b) Overlay of the noise-free IRF signal.

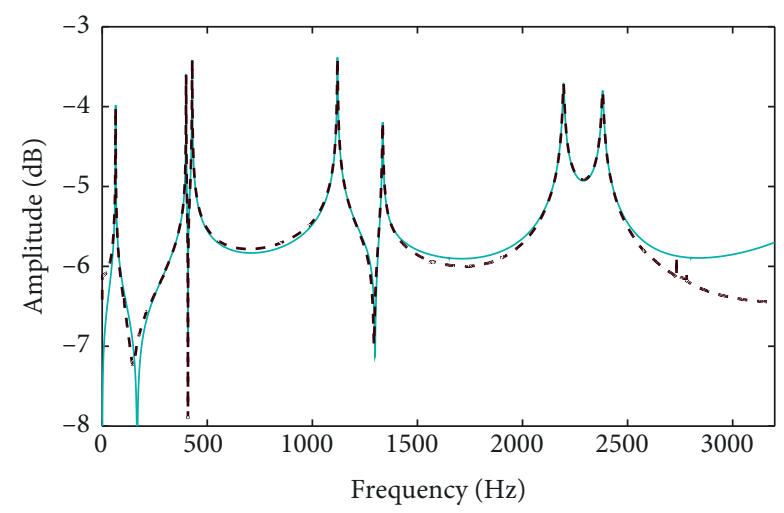

_ FRF signal from the noise-free IRF signal

- - - Noise-reduced FRF signal

(a)

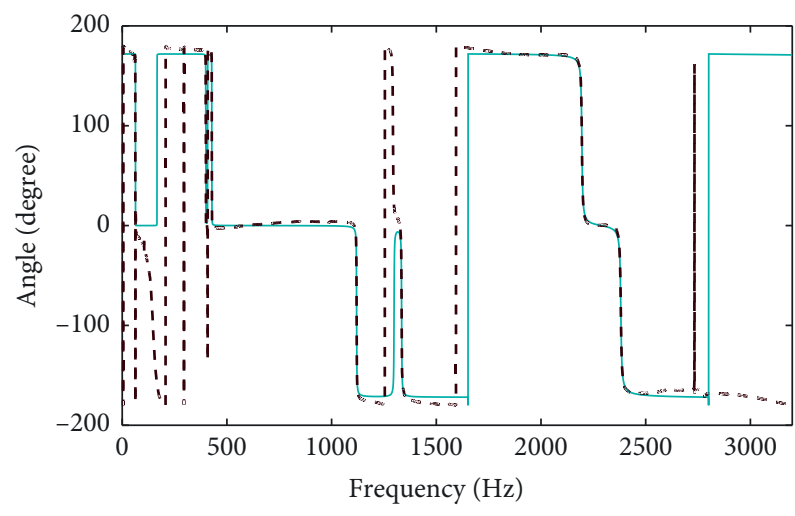

(b)

Figure 7: Noise reduction result of component signal reselection. (a) Energy spectrum. (b) Phase spectrum. 


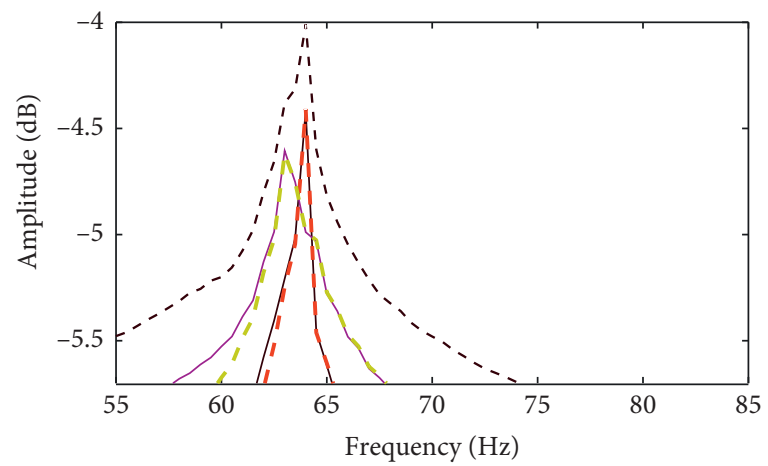

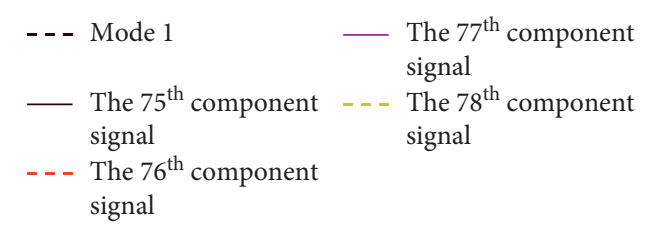

(a)
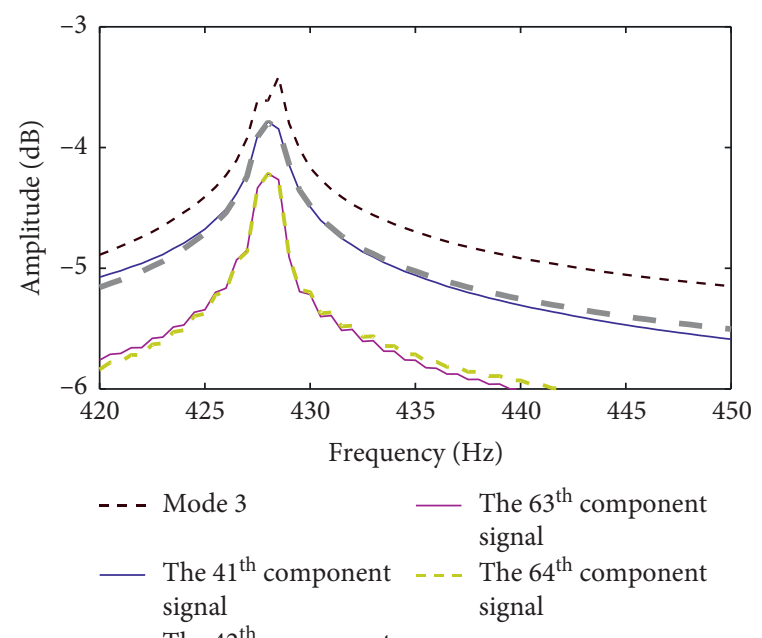

- - - The $42^{\text {th }}$ component signal

(c)

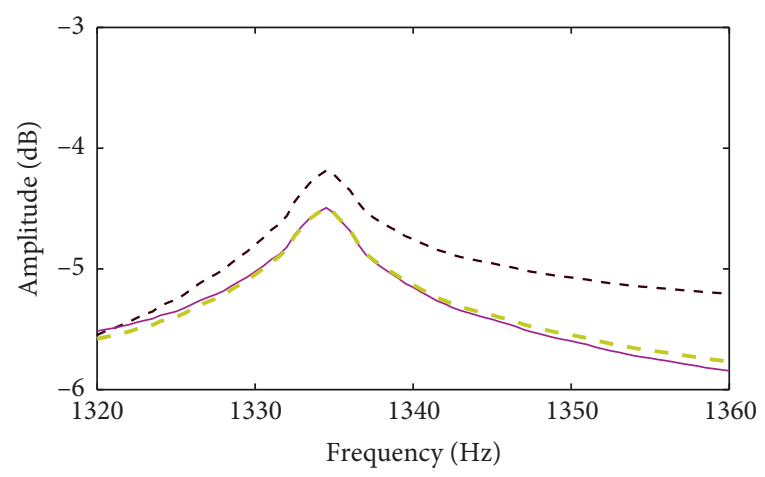

Mode 5

The $79^{\text {th }}$ component signal

The $80^{\text {th }}$ component signal

(e)

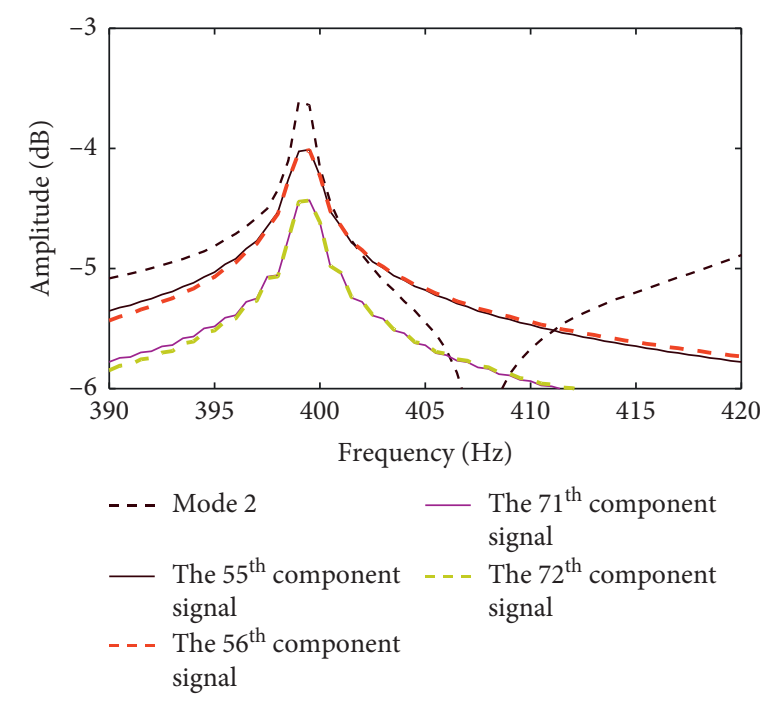

(b)

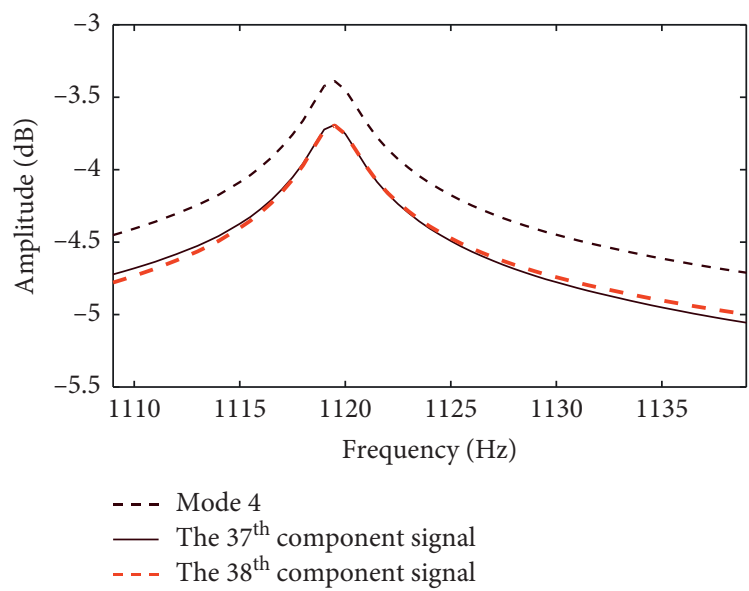

(d)

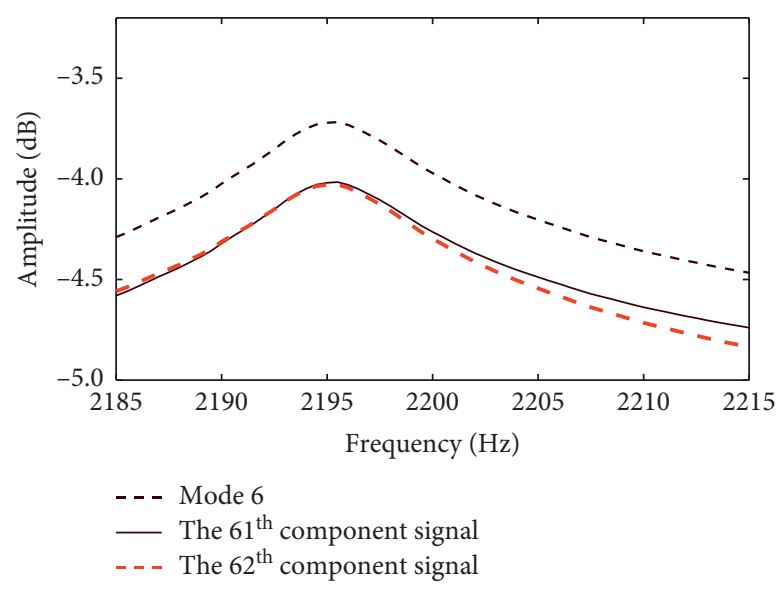

(f)

Figure 8: Continued. 


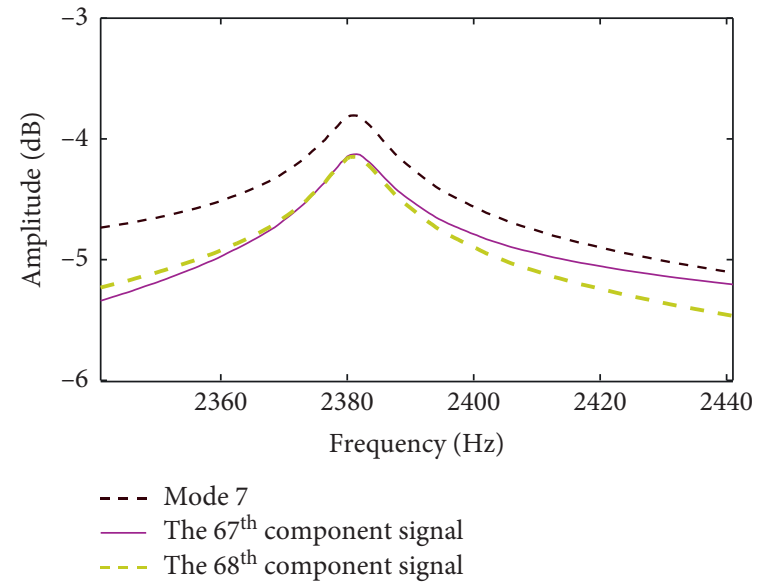

(g)

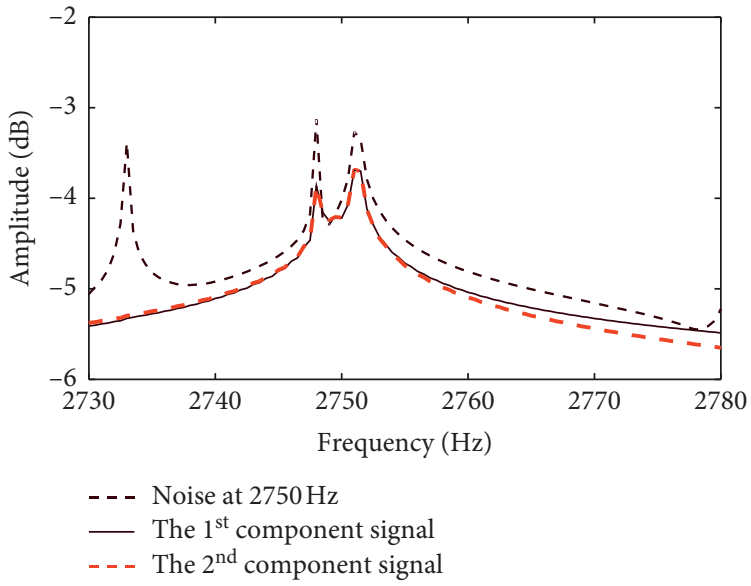

(h)

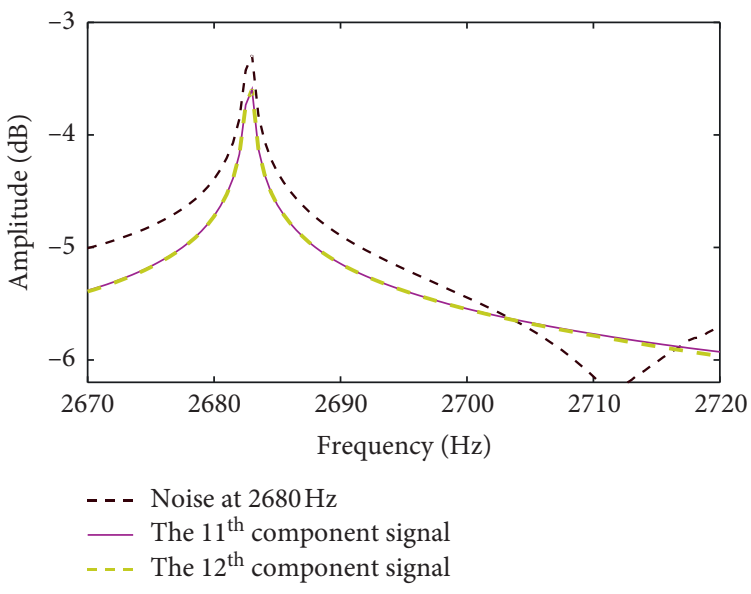

(i)

Figure 8: The modes and the corresponding component signals. (a) Component signals of mode 1 at $65 \mathrm{~Hz}$. (b) Component signals of mode 2 at $399 \mathrm{~Hz}$. (c) Component signals of mode 3 at $428 \mathrm{~Hz}$. (d) Component signals of mode 4 at $1119 \mathrm{~Hz}$. (e) Component signals of modal 5 at $1334 \mathrm{~Hz}$. (f) Component signals of modal 6 at $2195 \mathrm{~Hz}$. (g) Component signals of mode 7 at $2381 \mathrm{~Hz}$. (h) Component signals of noise at $2750 \mathrm{~Hz}$. (i) Component signals of the noise at $2572 \mathrm{~Hz}$.

component signals 61 and 62 . Mode 7 is formed by a pair of component signals 67 and 68. Then, Figures 8(h) and 8(i) give the energy spectra correlated with the harmonic noises. It can be found that the first two component signals belong to two harmonic noises near $2750 \mathrm{~Hz}$, since their peak frequencies are close, and their energy is similar. The pair of the $11^{\text {th }}$ and $12^{\text {th }}$ component signals forms a harmonic noise at $2572 \mathrm{~Hz}$. The peak frequencies of these noise component signals are not in the cared frequency bands, and they are abandoned as the noise.

The noise reduction result and the component signal analysis show that the reselection iteration with the bandwidth $35 \mathrm{~Hz}$ can separate the component signals of the modal modes from the component signals of the residue noises precisely. Then, in order to investigate the effect of the reselection with different pass bandwidths on denoising, the noise-reduced FRFs obtained from MPHSVD with bandwidths $2 \mathrm{~Hz}, 5 \mathrm{~Hz}, 10 \mathrm{~Hz}, 20 \mathrm{~Hz}, 45 \mathrm{~Hz}, 60 \mathrm{~Hz}$, and $80 \mathrm{~Hz}$ are plotted in Figure 9, with the overlay of the noise reduction result of the pass bandwidth $35 \mathrm{~Hz}$. It can be found that the results of the reselection of bandwidths $5 \mathrm{~Hz}, 10 \mathrm{~Hz}, 20 \mathrm{~Hz}$, $35 \mathrm{~Hz}, 45 \mathrm{~Hz}$, and $60 \mathrm{~Hz}$ are the same, which means the reselection iteration with the arbitrary bandwidth between $60 \mathrm{~Hz}$ and $5 \mathrm{~Hz}$ for the noised IRF here will achieve the same noise reduction quality. Then, when the bandwidth improves to $80 \mathrm{~Hz}$, one harmonic noise at $104.5 \mathrm{~Hz}$ is recovered. When the bandwidth decreases to $2 \mathrm{~Hz}$, the recovery of mode 1 at $65 \mathrm{~Hz}$ and mode 7 at $2381 \mathrm{~Hz}$ is incomplete. Thus, the reselection with too big bandpass width will recover some additional noise, and the reselection with too small bandpass width will lose some modal features. Then, since the least frequency interval between the modal peaks and the harmonic noise in the real modal test is unknown, it is difficult to decide the biggest frequency bandwidth that the residue noise will not be recovered. So, the frequency pass bandwidth of the reselection should be small to avoid the recovery of the noise, but not too small that the modal features are abandoned. One frequency bandwidth like $5 \mathrm{~Hz}$ or $10 \mathrm{~Hz}$ is enough for the recovery of the whole modal modes and the removal of the noise. 

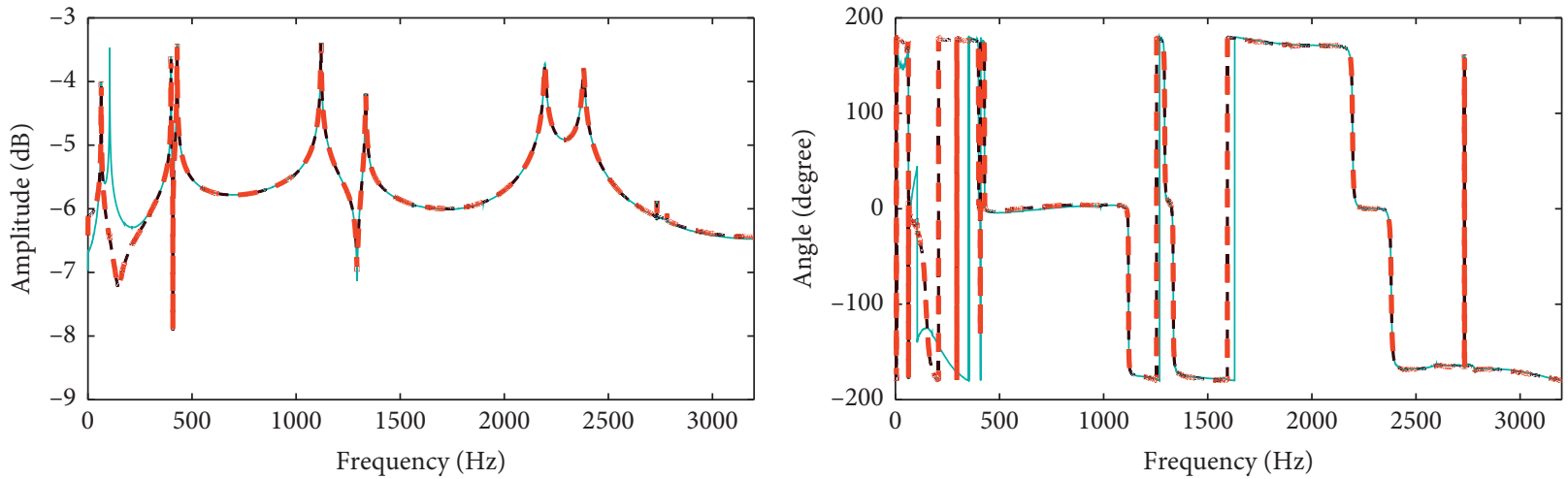

The result of the $80 \mathrm{~Hz}$ pass bandwidth

- - - The result of the $60 \mathrm{~Hz}$ pass bandwidth

- - - The result of the $45 \mathrm{~Hz}$ pass bandwidth

(a)
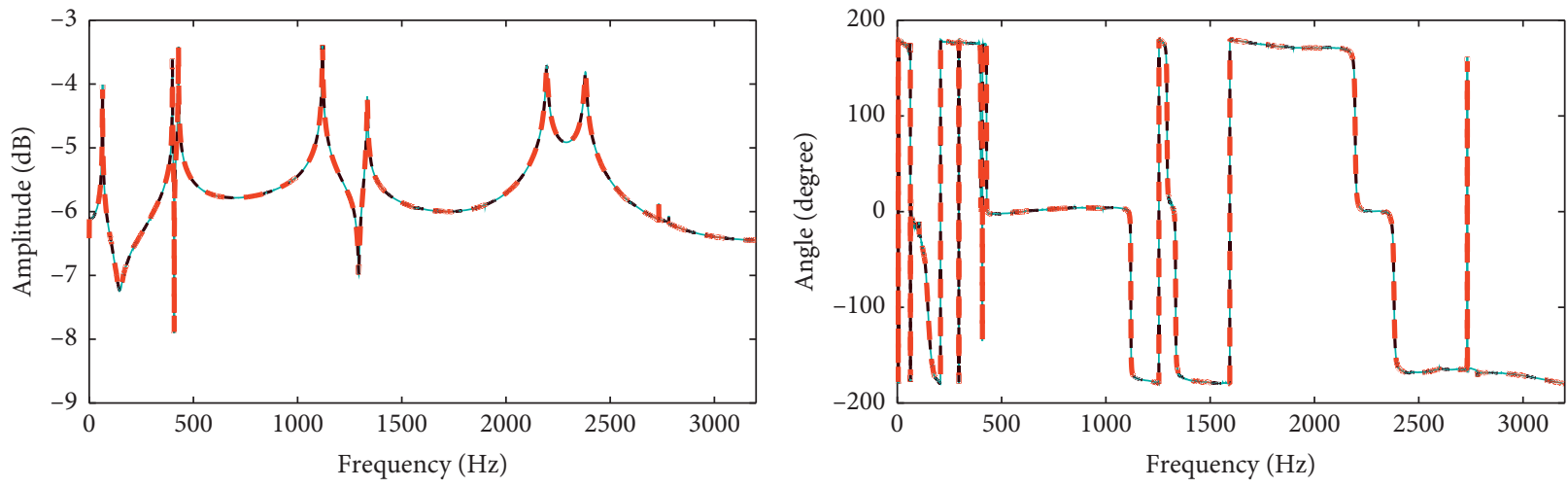

_ The result of the $35 \mathrm{~Hz}$ pass bandwidth

- - - The result of the $30 \mathrm{~Hz}$ pass bandwidth

- - - The result of the $20 \mathrm{~Hz}$ pass bandwidth

(c)
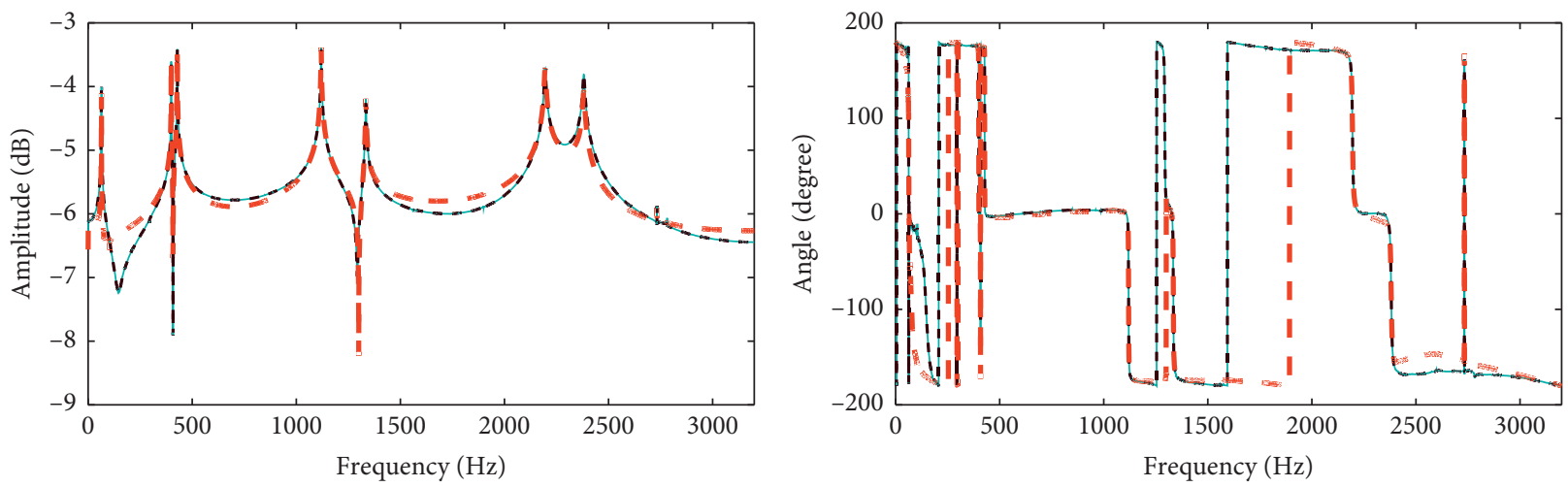

- The result of the $10 \mathrm{~Hz}$ pass bandwidth

- - - The result of the $5 \mathrm{~Hz}$ pass bandwidth

- - - The result of the $2 \mathrm{~Hz}$ pass bandwidth

(e)

(f)

Figure 9: The results with different pass bandwidths. (a, c, e) Energy spectrum comparison. (b, d, f) Phase spectrum comparison.

3.4. Modal Parameter Identification with the Noise-Reduced FRF. After the MPHSVD is implemented, the curve fitting with the rational fraction polynomial (RFP) method is conducted to extract the modal parameters. Modal modes are identified piecewise, and the modal frequencies with the correlated damping factors are extracted for each mode. The curving fitting of the noise-reduced FRF signal is plotted in Figure 10, and the identified modal parameters are listed in Table 2. From Figure 10, the fitted curve fits the noise-reduced FRF signal well, which shows the validity of curve 


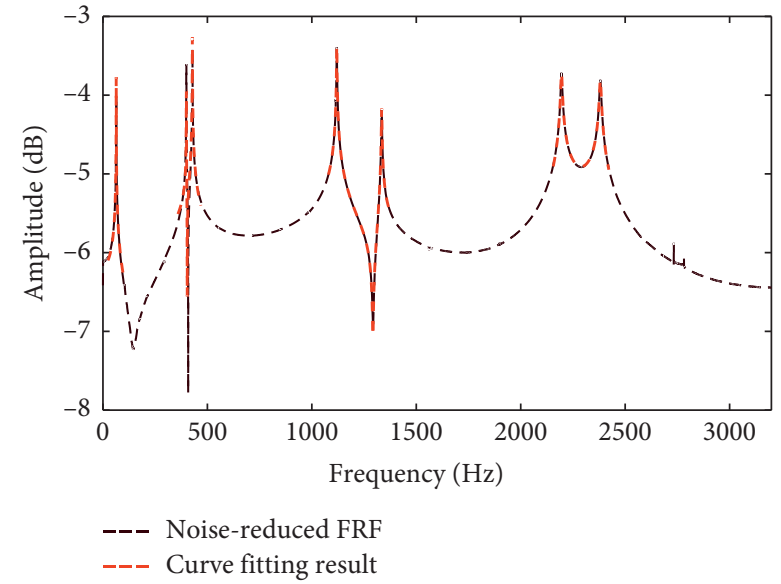

(a)

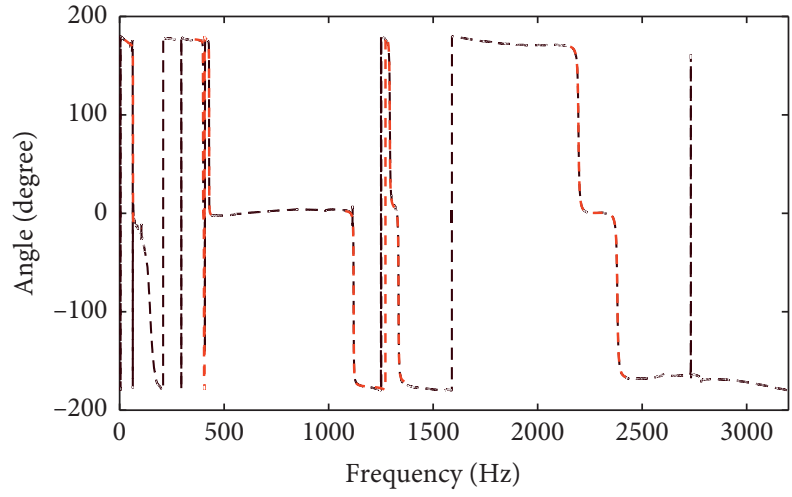

(b)

FIGURE 10: Curve fitting result of the noise-reduced FRF signal. (a) Curve fitted energy spectrum of denoised FRF. (b) Curve fitted phase spectrum of denoised FRF.

TABLE 2: Modal parameters of the noised and noise-reduced FRF signals.

\begin{tabular}{lcccccc}
\hline Mode & $\begin{array}{c}\text { Modal frequency } \\
(\mathrm{Hz})(\text { original) }\end{array}$ & $\begin{array}{c}\text { Modal frequency } \\
(\mathrm{Hz})(\text { denoised) }\end{array}$ & $\begin{array}{c}\text { Frequency } \\
\text { difference }(\mathrm{Hz})\end{array}$ & $\begin{array}{c}\text { Damping factor } \\
\text { (original) }\end{array}$ & $\begin{array}{c}\text { Damping factor } \\
(\text { denoised) }\end{array}$ & $\begin{array}{c}\text { Damping factor } \\
\text { difference }(\%)\end{array}$ \\
\hline 1 & 63.6779 & 63.61561 & 0.062331 & 0.000674 & 0.001091 & 61.78994 \\
2 & 399.332 & 399.2953 & 0.036889 & 0.000295 & 0.000393 & 32.93652 \\
3 & 428.076 & 428.108 & 0.032425 & 0.000667 & 0.000553 & 17.06583 \\
4 & 1119.44 & 1119.429 & 0.013394 & 0.000866 & 0.000788 & 8.984838 \\
5 & 1334.57 & 1334.775 & 0.202722 & 0.000943 & 0.000892 & 5.423373 \\
6 & 2195.12 & 2195.505 & 0.385319 & 0.001474 & 0.001456 & 1.267132 \\
7 & 2381.41 & 2380.844 & 0.562656 & 0.001588 & 0.001563 & 1.541009 \\
\hline
\end{tabular}

fitting. From Table 2, it can be seen that there is little difference between identified modal frequencies and the original modal frequencies, which is more precise than the modal frequencies extracted from the noised FRF signal. Then, compared with the modal damping factors from the noised FRF signal, the damping factor of mode 1 is positive, and the difference between the damping factors of mode 1 significantly decreases from $1100 \%$ to $60 \%$. In practical applications, the first mode is usually considered as the fundamental frequency, and the $60 \%$ difference of the damping factor still looks high. The better way to deal with it maybe is to pick up another measurement location in order to increase the energy of mode 1 . The damping factor of mode 2 from the noise-reduced FRF signal is much closer to the correlated damping factor of the noise-free FRF signal, where the difference of damping factors of mode 2 decreases from $160 \%$ to $32 \%$. The other damping factors extracted from the noise-reduced FRF signal are also closer to the original damping factors extracted from the noisefree FRF signal compared with those extracted from the noised FRF signal. Thus, the analysis shows that the curve fitting of the noise-reduced FRF signal is precise, and the modal parameters extracted from the noise-reduced FRF signal are more precise than those from the noised FRF signal, which further proves that the noise reduction is successful.

In order to further investigate the effectiveness of the MPHSVD to deal with various degrees of the noise, four degrees of the random Gaussian noise, listed as 3\%, 5\%, 7\%, and $9 \%$ ratios between the amplitudes of the added Gaussian noise and the max amplitude of the noise-free IRF signal, respectively, are added to the simulated noise-free IRF signal. Five simulations of each ratio are conducted, and their corresponding mean SNRs are $7.68 \mathrm{~dB}, 3.25 \mathrm{~dB}, 0.31 \mathrm{~dB}$, and $-1.89 \mathrm{~dB}$, respectively. Then, the noise removal rates are calculated by equation (22), and the results with the corresponding noise reduction successful rate are shown in Table 3. It shows that as the noise amplitude increases, the noise removal rate decreases and the noise reduction successful rate also decreases. The overlays of the noise-reduced FRF signals against the noised FRF signals of the simulations given in italics in Table 3 are conducted, and the result is shown in Figure 11.

Figures 11(a) and 11(b) show that the noise removal rates can reach over $90 \%$ when SNRs of the noising FRFs are $7.68 \mathrm{~dB}$ and $3.25 \mathrm{~dB}$, respectively. The noise removal rate decreases when the SNR becomes worse. In Figure 11(d), the FRF in green color contaminated with the heaviest noise with an SNR of $-1.89 \mathrm{~dB}$ can hardly distinguish the vibration modes. After reducing the noise, most of concerned modes in black color except for mode 5 can be clearly seen although the noise-reduced FRF looks a bit rougher than the noise-free FRF.

\section{Case Study II: Experiment}

4.1. Experiment Configuration. To further verify the proposed method, an experimental test is undertaken. The force 
TABLE 3: Noise reduction result.

\begin{tabular}{lcccc}
\hline Simulation time & $\begin{array}{c}\text { Noise removal rate } \\
(\text { SNR }=7.68 \mathrm{~dB})\end{array}$ & $\begin{array}{c}\text { Noise removal rate } \\
(\text { SNR }=3.25 \mathrm{~dB})\end{array}$ & $\begin{array}{c}\text { Noise removal rate } \\
(\mathrm{SNR}=0.31 \mathrm{~dB})\end{array}$ & $\begin{array}{c}\text { Noise removal rate } \\
(\mathrm{SNR}=-1.89 \mathrm{~dB})\end{array}$ \\
\hline 1 & 0.947717 & 0.902006 & 0.858859 & - \\
2 & 0.945414 & 0.903764 & 0.881271 & 0.845278 \\
3 & 0.941209 & 0.916029 & - & 0.837777 \\
4 & 0.942136 & 0.916785 & - & 0.776679 \\
5 & 0.947392 & 0.906086 & $60 \%$ & $60 \%$ \\
Successful rate & $100 \%$ & $100 \%$ & & 0.815477 \\
\hline
\end{tabular}

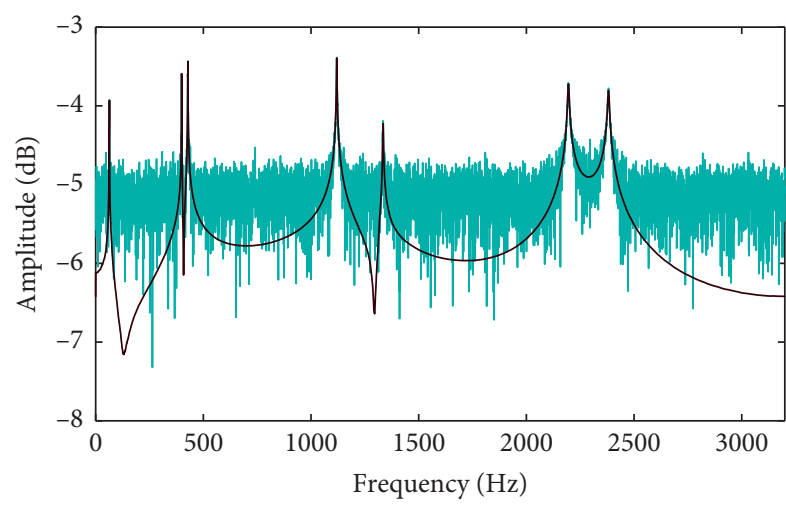

- Noised FRF signal of SNR $=7.6848 \mathrm{~dB}$

— Noise-reduced FRF signal

(a)

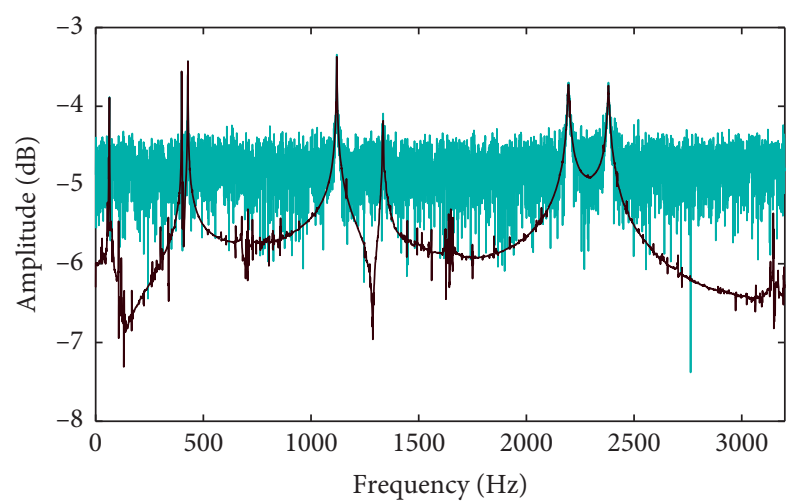

— Noised FRF signal of SNR $=0.3107 \mathrm{~dB}$

— Noise-reduced FRF signal

(c)

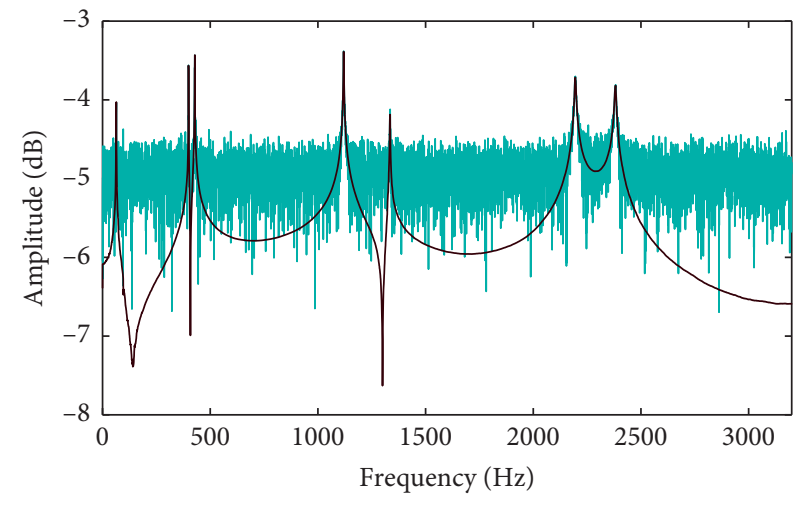

- Noised FRF signal of SNR $=3.2554 \mathrm{~dB}$

- Noise-reduced FRF signal

(b)

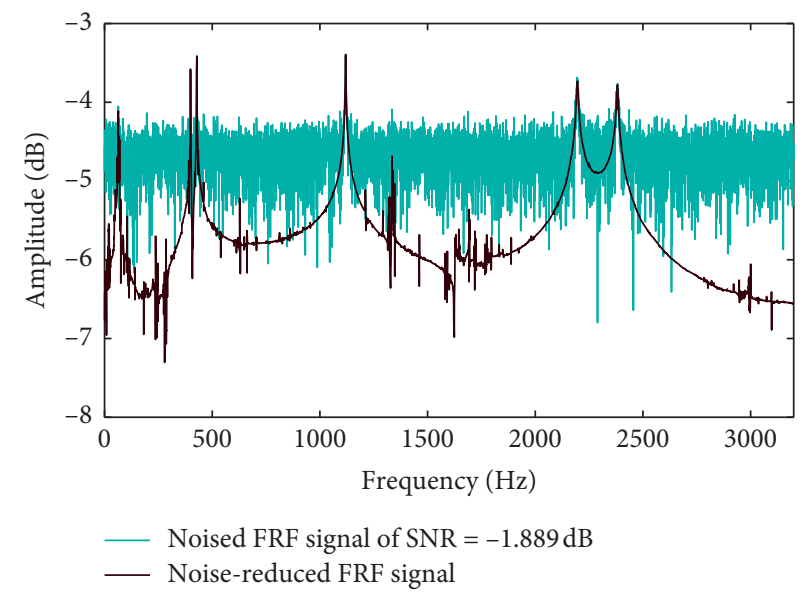

(d)

FIGURE 11: Overlays of the FRFs with various SNR values. (a) Overlay of the FRFs with $\mathrm{SNR}=7.68 \mathrm{~dB}$. (b) Overlay of the FRFs with $\mathrm{SNR}=3.25 \mathrm{~dB}$. (c) Overlay of the FRFs with $\mathrm{SNR}=0.31 \mathrm{~dB}$. (d) Overlay of the FRFs with $\mathrm{SNR}=-1.89 \mathrm{~dB}$.

hammer typed as PCB086C03, manufactured by PCB, is used for excitation. The UM-40-AB-HF electromagnetic heater and the heating coil, produced by UIHM, are used for heating the plate. The 3D scanning laser Doppler vibrometers (SLDVs) typed with PSV-400-3D for response measurement are manufactured by Polytec. The experimental setup is shown in Figure 12(a), and the measuring points are shown in Figure 12(b).

In the modal test, the flat plate is firstly grounded at the one end to a metal baffle tightened with four bolts, and the baffle is connected to the test bench to monitor the rigid support. Then, the heating coil is connected to the bench behind the plate and connected to the electromagnetic heater for heating the plate. The force hammer is then connected to the computer for excitation and measuring the input force. Subsequently, the SLDVs are placed in front of the test plate for the noncontact measurement. The measuring points are evenly distributed on the plate, and totally 45 points are selected.

The test starts when the temperature of the plate is heated and stabled at the 200 centigrade degrees. The analysis frequency range is $6400 \mathrm{~Hz}$, and it takes 2 seconds once for each measuring point. After testing, the FRF signals are obtained for these 45 measuring points. 


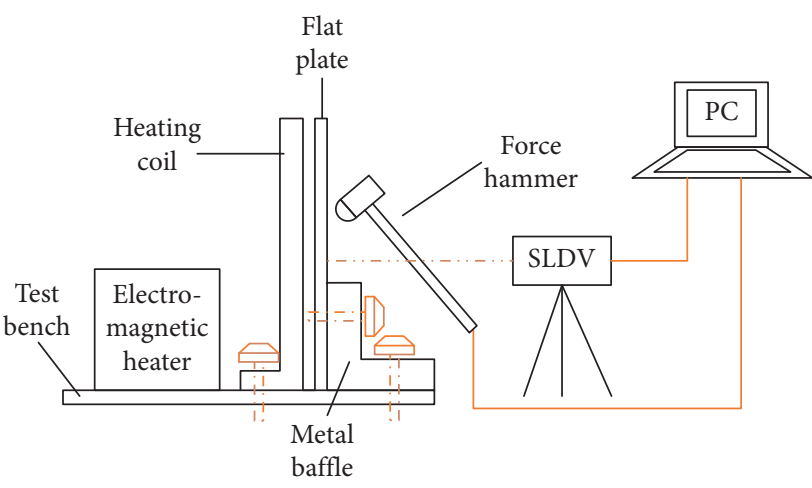

(a)

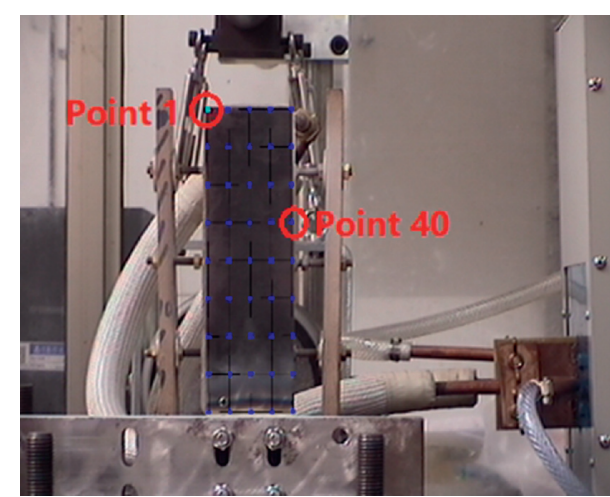

(b)

FIgURE 12: (a) The experimental setup. (b) The measurement points.

4.2. The Analysis of the Noised FRF Signals. The measured FRF signals of Point 40 and Point 1, marked as the red circle in Figure 12(b), at 200 centigrade degrees are taken as the example. The overlay of the energy and the phase spectra of the FRF signals of Points 1 and 40 in the heated temperature is plotted in Figure 13.

It can be found from Figure 13(a) that the energy spectrum of Point 1 is clearer, but the spectrum of Point 40 contains heavy noise and cannot be directly used for modal parameter identification. One contributing factor is thought that the speckle noise of the FRF signal is enhanced by the high temperatures, since the speckle noise is usually dominant in the measurement of the SLDV [31]. The ten peak amplitudes and frequencies in the red and blue circles are the ten modal modes we concerned about. These peak frequencies are selected from the clear energy spectrum of point 1 by observation. The modal peaks in the blue circles can only be observed in the energy spectrum of Point 1, and the correlated modal peaks of Point 40 are totally drown into the noise, which means they cannot be recovered by the MPHSVD filter. Thus, the modal peak frequencies and amplitudes of the red circles in the light blue energy spectrum are the concerned modal features of Point 40 and those of the whole red and blue circles in the black curve are the concerned modal features of Point 1 . The other peaks in the signal are thought to be induced by the added mass of the vibrator, and they are not selected for recovery Figure 13(b) gives the phase spectra of the two FRF signals. Similarly, the phase spectrum of Point 40 contains heavy noise, and some of the modal phase changes cannot be seen clearly.

4.3. The Noise Reduction of the Measured FRF Signal by the MPHSVD Method. For the transformed IRF signals of Point 40 and Point 1, the MPHSVD is conducted to eliminate the noise and retain the concerned modal features. The amplitude difference threshold of accumulation is set as 0.15 , and the component signal accumulations of the two transformed IRF signals are conducted. The accumulation of the transformed IRF signal of Point 1 stops at the 43 iteration turn, and the first 43 component signals which cover the whole concerned modal modes are selected. Then, as there are ten modal peaks selected from the energy spectrum of IRF signal 1, ten frequency pass bands with the bandwidth $10 \mathrm{~Hz}$ near the modal peak frequencies are set for the reselection of the component signals of the modal modes. Finally, 32 component signals of the first 43 component signals are reselected, and they are added up to form the noise-reduced IRF signal and the noise-reduced FRF signal. For the transformed IRF signal of Point 40, the first 1119 component signals are selected in the accumulation. Then, as there are 8 modal peaks selected from IRF 40, eight frequency bands with the bandwidth $10 \mathrm{~Hz}$ are set for the component signal reselection, and 25 component signals from the first 1119 component signals of IRF 40 are reselected for the construction of the noise-reduced FRF signal. Finally, the overlay between the denoised FRF signals and the FRF signals from the transformed IRF signals is given in Figure 14.

From Figures 14(a) and 14(b), the noise reduction result of Point 1 can be seen. It can be seen that the concerned modes are retained, and the spectra of the energy and phase are much smoother and clearer after noise reduction, which shows the validity of the noise reduction method. Figures $14(\mathrm{c})$ and 14(d) give the noise reduction result of Point 40. It shows that the spectra are much smoother than the original spectra of the transformed IRF signal, and most concerned modal modes are recovered completely. However, the amplitudes of the modal modes at 1127 and $1149 \mathrm{~Hz}$ are not recovered completely, and the modal mode at $1014 \mathrm{~Hz}$ is nearly eliminated after the noise reduction, which is marked in the red circles in Figure 14(a). The main reason is that these modes are contaminated with the noise heavily, and their correlated component signals are aliased with the noise. The modes at 1014, $1127 \mathrm{~Hz}$, and $1149 \mathrm{~Hz}$ with their correlated decomposed component signals are shown in Figures 15 and 16. Figure 15(a) gives the energy spectra of modal mode at 1127 and $1149 \mathrm{~Hz}$, and it is obvious that these modal modes are contaminated with the noise heavily, since their amplitudes fluctuate in the whole observed frequency range. Figure 15(b) gives the noise mode at 


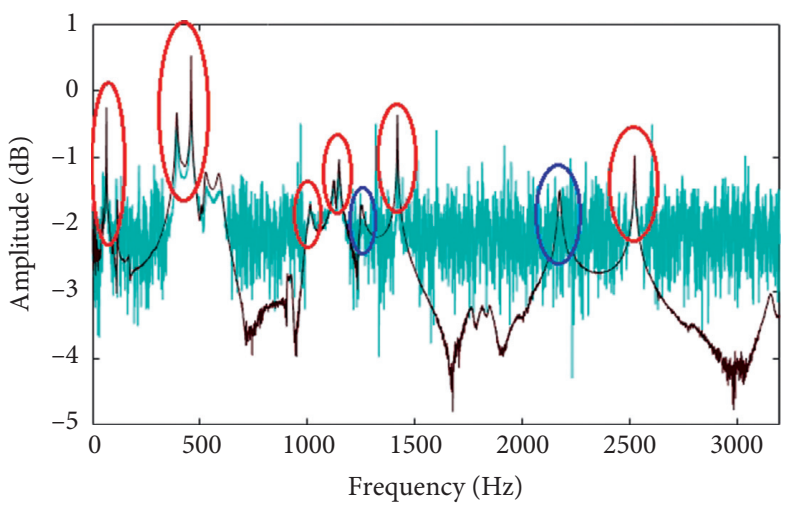

- FRF signal of point 40 at 200 degrees FRF signal of point 1 at 200 degrees

(a)

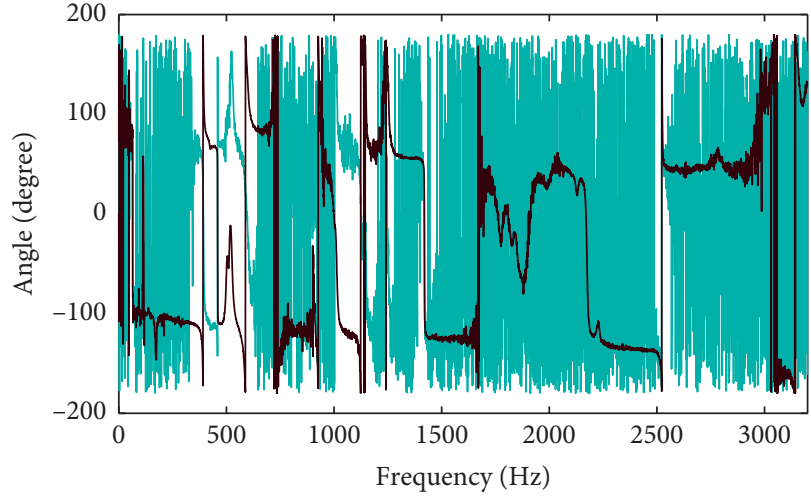

(b)

Figure 13: Overlay of the FRFs of Point 1 and Point 40. (a) Energy spectrum comparison. (b) Phase spectrum comparison.

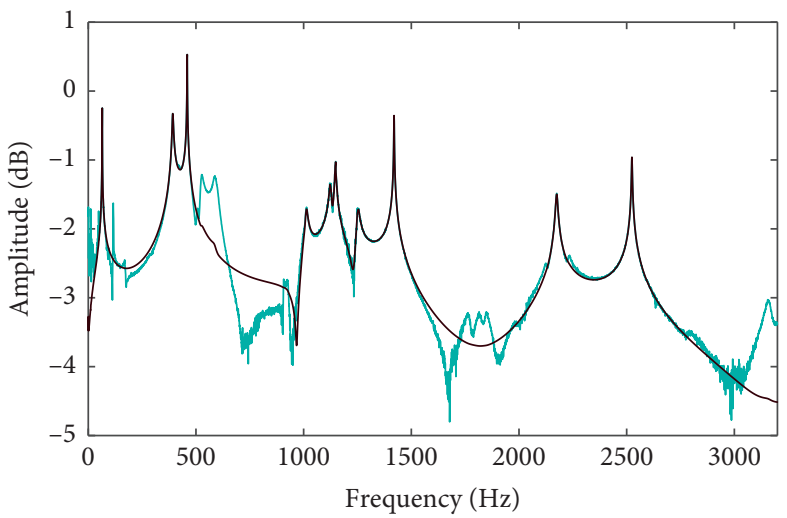

_ FRF signal from IRF1

_ Noise-reduced FRF signal of point 1

(a)

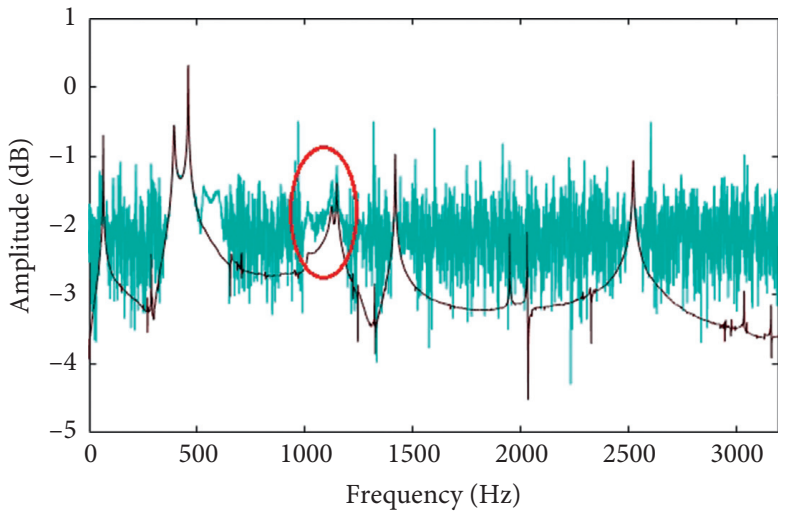

— Noise-reduced FRF signal of point 40

(c)

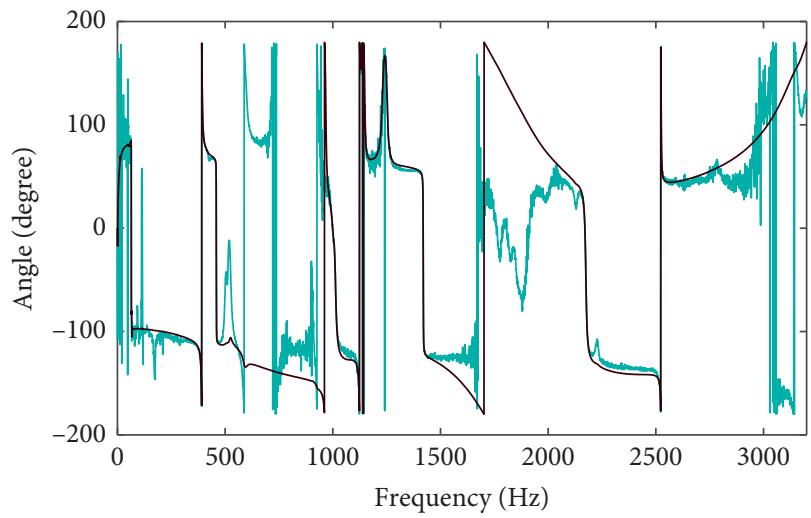

(b)

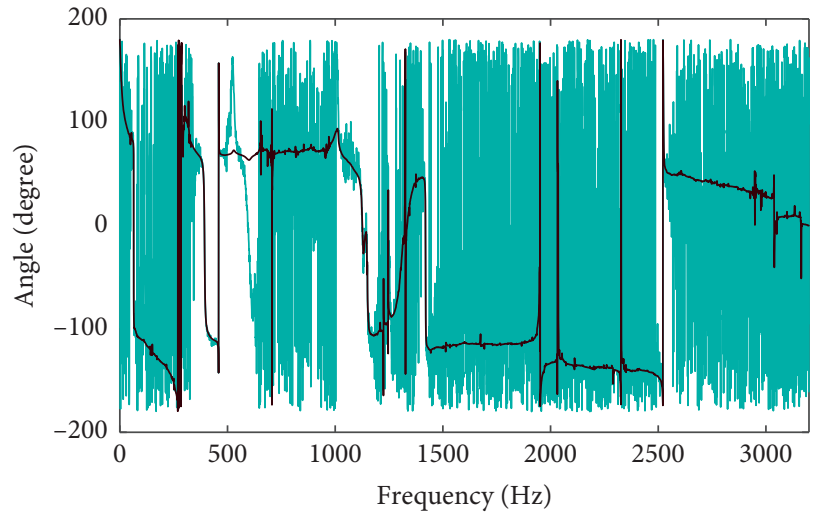

(d)

FIgure 14: Noise reduction result of Points 1 and 40. (a) Energy spectrum of Point 1. (b) Phase spectrum of Point 1. (c) Energy spectrum of Point 40. (d) Phase spectrum of Point 40. 


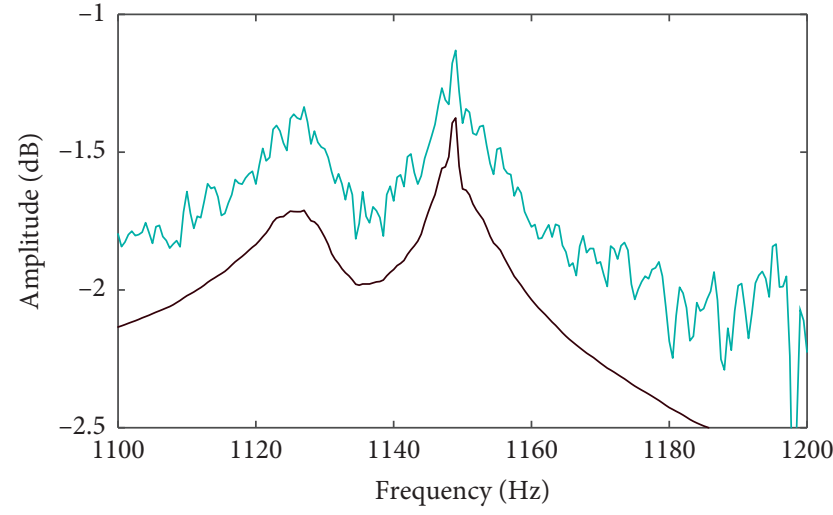

Noise mode at $1127,1149 \mathrm{~Hz}$

Denoised mode

(a)

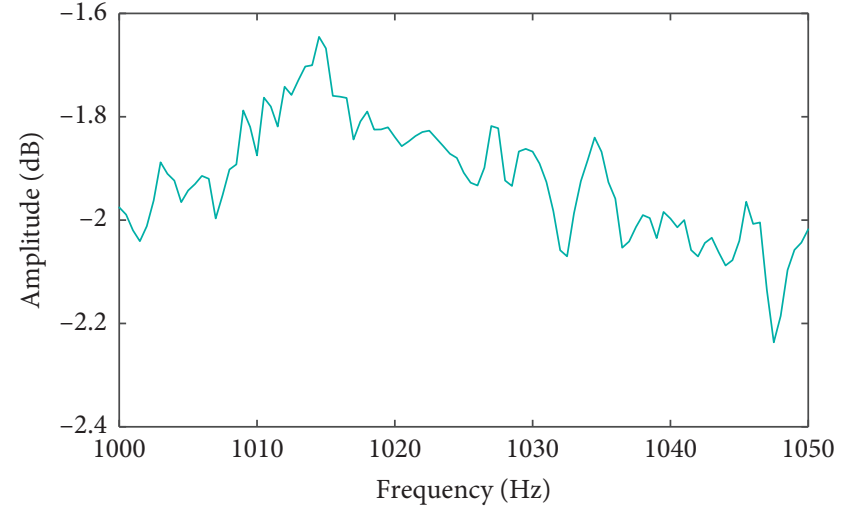

_ Noise mode at $1014 \mathrm{~Hz}$

Figure 15: (a) Noise modes at 1127 and $1149 \mathrm{~Hz}$. (b) Noise mode at $1014 \mathrm{~Hz}$.

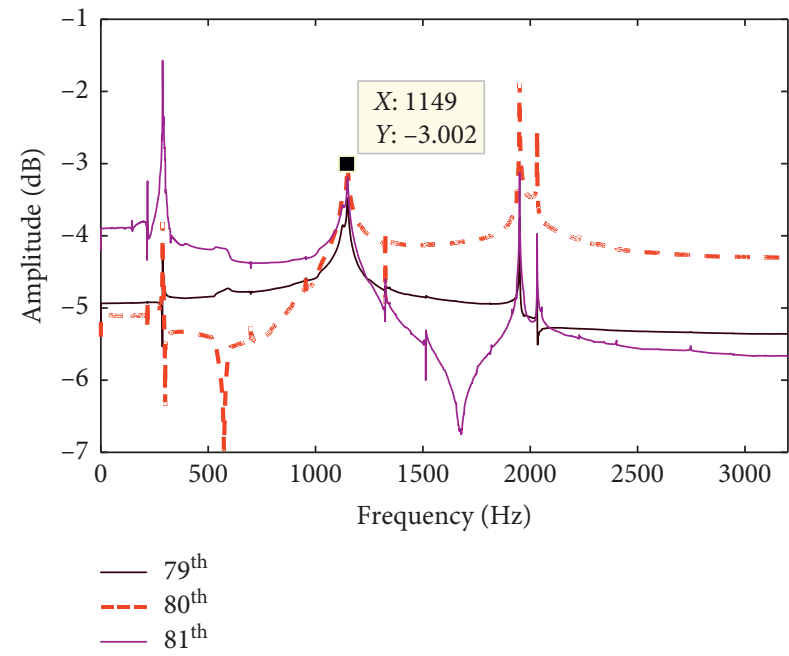

(a)

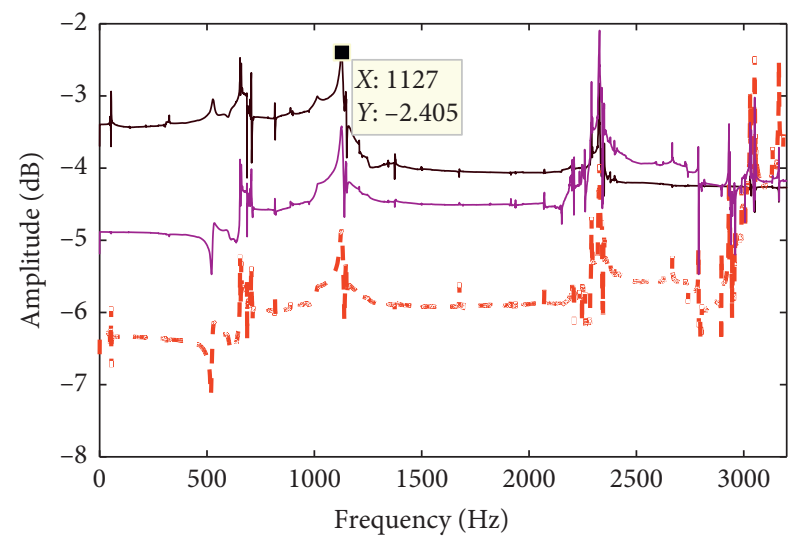

$-275^{\text {th }}$
$---276^{\text {th }}$

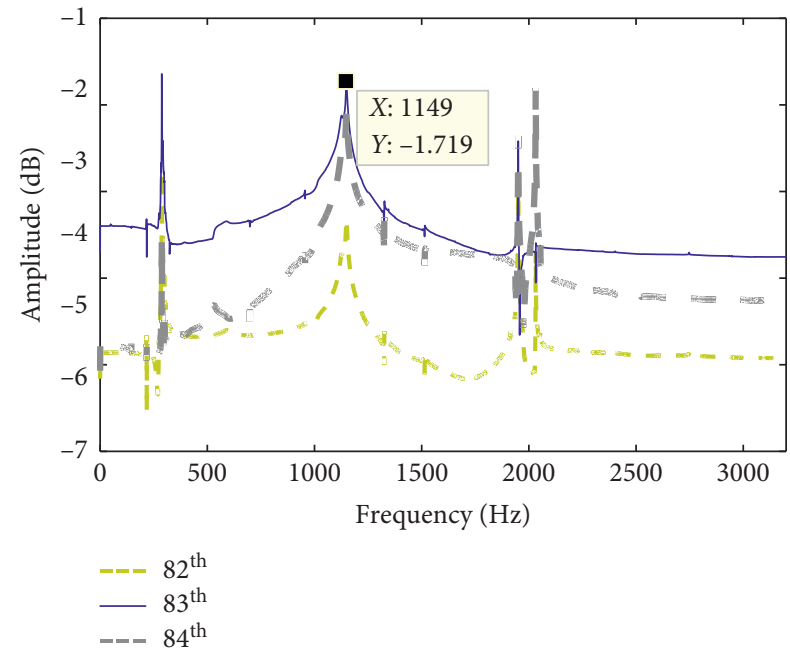

(b)

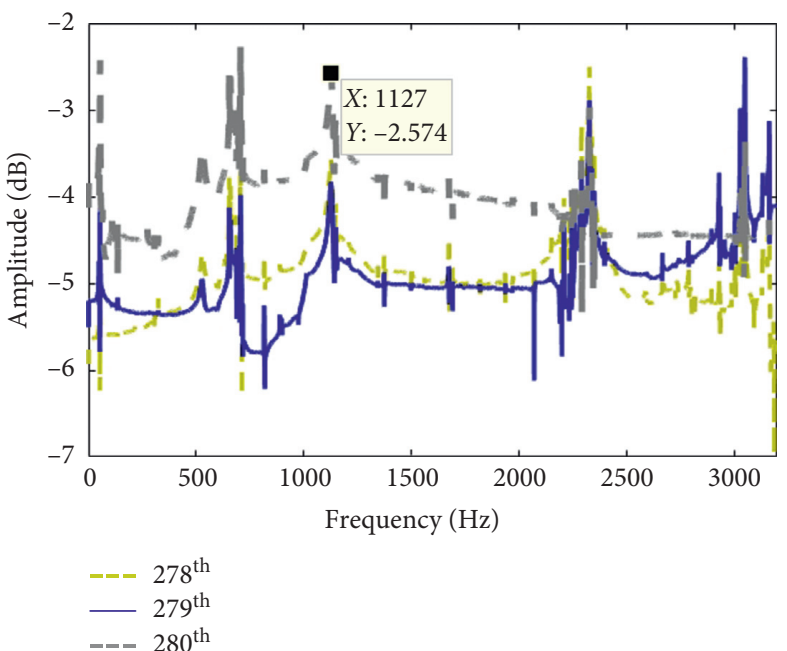

(d)

FIgURE 16: Continued. 

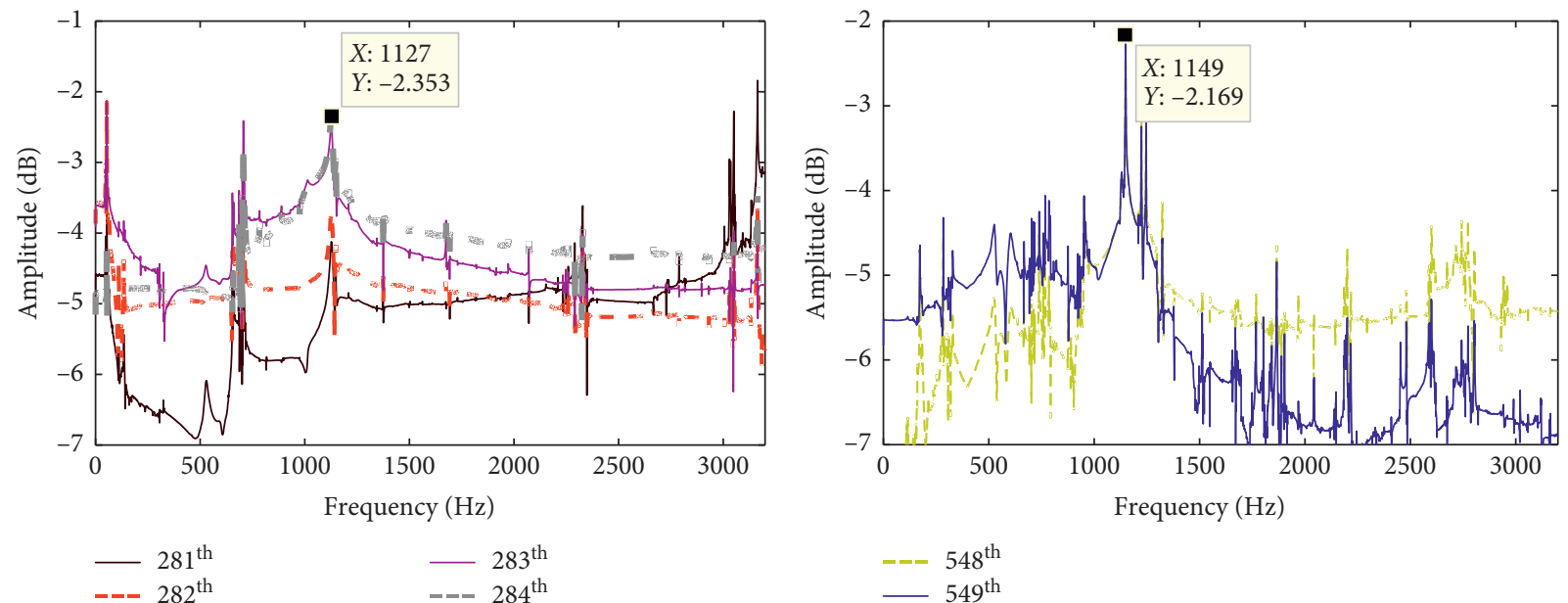

(e)

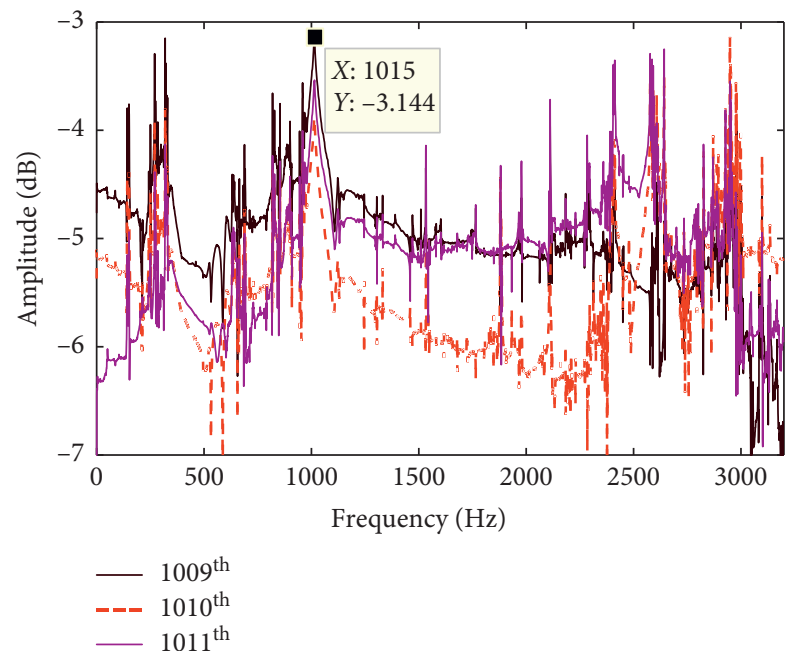

(f)

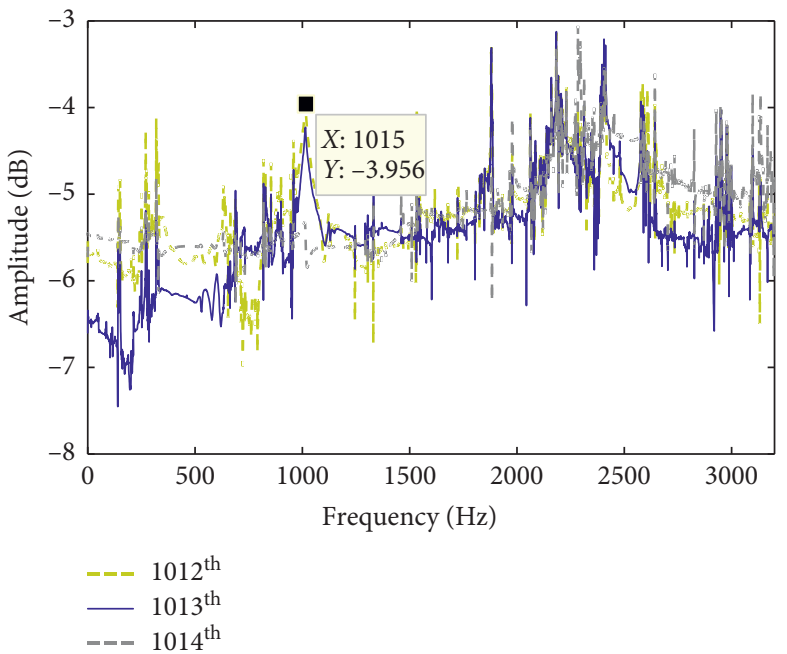

(g)

(h)

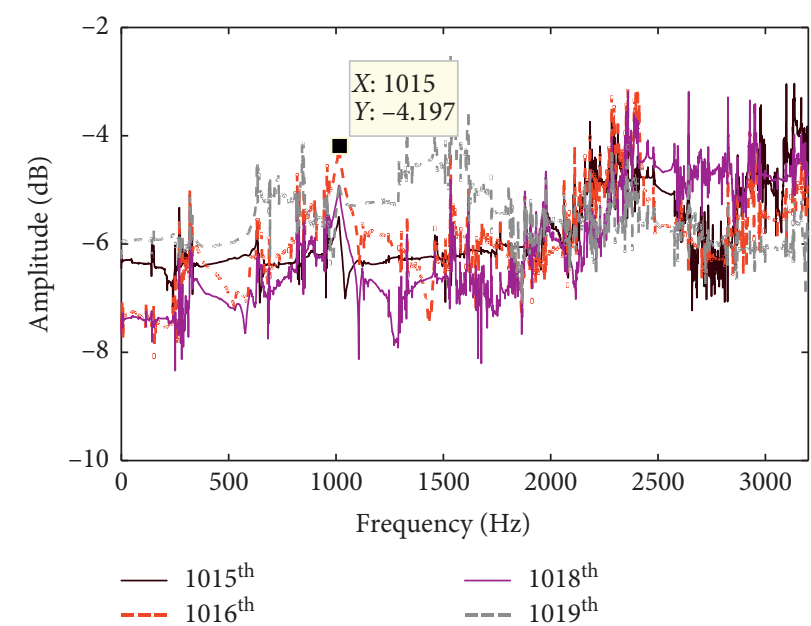

(i)

FIGURE 16: Component signals of noise modes at 1014, 1127, and $1149 \mathrm{~Hz}$. (a) Component signals 78, 80, and 81. (b) Component signals 79, 80, and 81. (c) Component signals 275, 276, and 277. (d) Component signals 278, 279, and 280. (e) Component signals 281, 282,283 , and 284. (f) Component signals 548 and 549. (g) Component signals 1009, 1010, and 1011. (h) Component signals 1012, 1013, and 1014. (i) Component signals 1015, 1016, 1018, and 1019. 


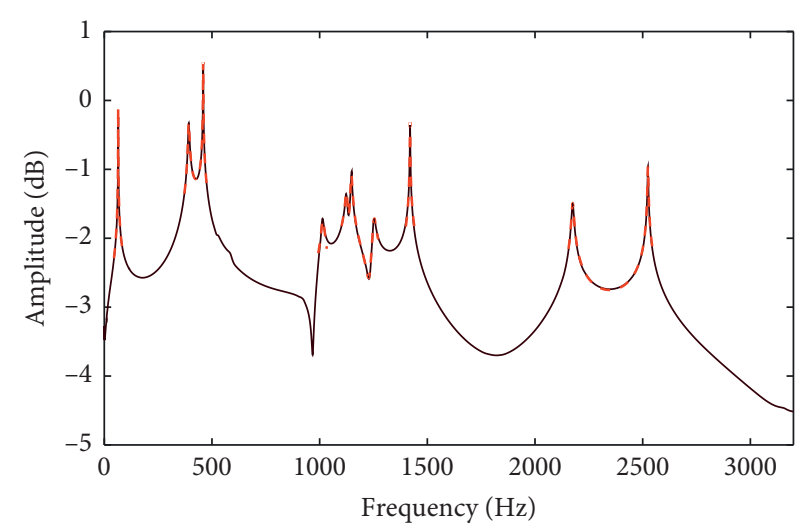

— Noise-reduced FRF of point 1 - - - Curve fitting result

(a)

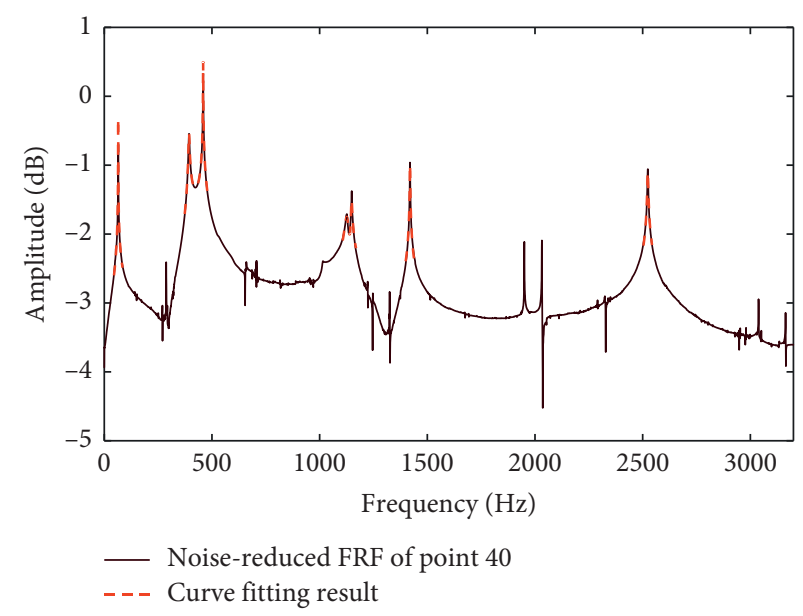

(c)

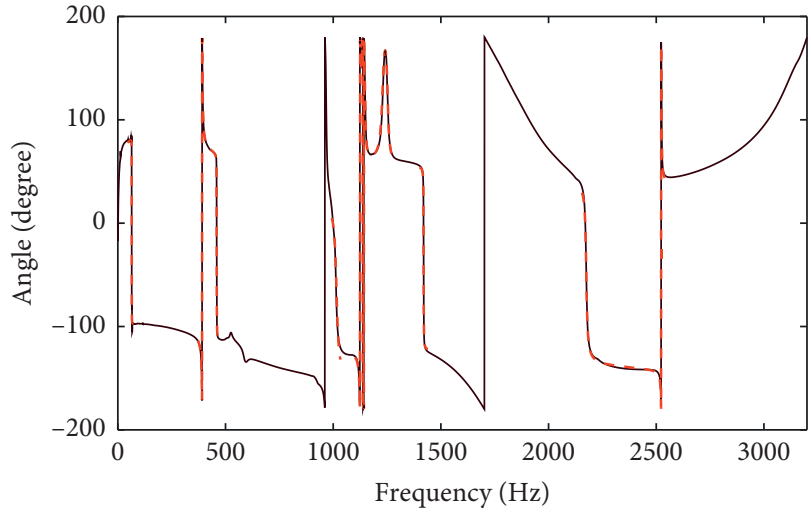

(b)

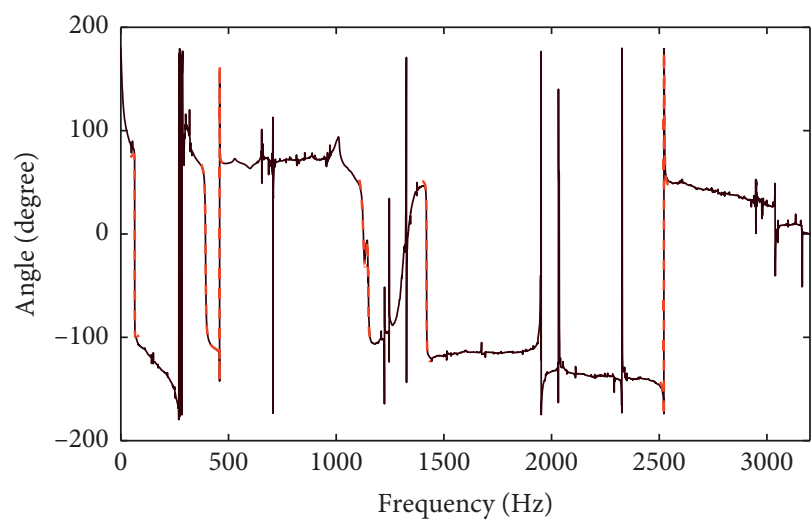

(d)

Figure 17: Curve fit of the noise-reduced FRF signal. (a) Energy spectrum of Point 1. (b) Phase spectrum of Point 1. (c) Energy spectrum of Point 40. (d) Phase spectrum of Point 40.

$1014 \mathrm{~Hz}$, and the figure shows that this mode is noisier than modes at 1127 and $1149 \mathrm{~Hz}$, where it is hard to see the modal behavior from the spectrum. The decomposed component signals correlated with the three modes are shown in Figures 16(a). The mode at $1149 \mathrm{~Hz}$ is decomposed to the component signals from 79 to 84 and component signals 548 to 549 . The mode at $1127 \mathrm{~Hz}$ is decomposed to the component signals from 275 to 284 . The mode at $1014 \mathrm{~Hz}$ is decomposed to the component signals 1009 to 1016 and component signals 1018 to 1019 . Because of the heavy noise with the modes, these decomposed component signals are aliased with the noise heavily. Besides, for the most decomposed component signals of the three modes, the peak amplitude of the noise is bigger than the peak amplitude correlated with the modal modes, which means the noise energy is bigger than the modal modes. Thus, most of the component signals correlated with the three modes are abandoned as the noise, and only a few of them are used for recovery. For the mode at $1149 \mathrm{~Hz}$, the reselected component signals are component signals 79,548 , and 549, while the other component signals from 80 to 84 are abandoned. The reselected component signals of the mode at $1127 \mathrm{~Hz}$ are component signals 275 and 284, and the component signals from 276 to 283 are abandoned. The reselected component signal of the mode at $1014 \mathrm{~Hz}$ is the component signal 1009 , while the other 9 component signals correlated with the mode are eliminated. Therefore, the amplitudes of the modes at 1127 and $1149 \mathrm{~Hz}$ are not recovered completely, and the mode at $1014 \mathrm{~Hz}$ is nearly eliminated.

\subsection{Modal Identification of the Noise-Reduced Measured FRF} Signal. After the MPHSVD is done, the RFP method is conducted to extract the modal parameters of the two denoised FRF signals. The curve fitting result and the extracted modal parameters of the concerned modes are shown in Figure 17 and Table 4. Figure 17 gives the curve fitting result of the two noise-reduced FRF signals. It can be found that the modal modes are fitted well, and the fitted curves have a good coincidence with the denoised FRF signals. Besides, the mode at $1014 \mathrm{~Hz}$ of Point 40 is nearly eliminated since it is contaminated with the noise heavily, and it is not fitted to extract the modal parameters. Table 4 gives the extracted modal parameters of the two denoised 
TABLE 4: The extracted modal parameters.

\begin{tabular}{|c|c|c|c|c|c|c|}
\hline Mode & $\begin{array}{l}\text { Modal frequency } \\
(\mathrm{Hz}) \text { (Point } 1)\end{array}$ & $\begin{array}{l}\text { Modal frequency } \\
\text { (Hz) (Point 40) }\end{array}$ & $\begin{array}{c}\text { Frequency } \\
\text { difference }(\mathrm{Hz})\end{array}$ & $\begin{array}{l}\text { Damping factor } \\
\text { (Point } 1)\end{array}$ & $\begin{array}{l}\text { Damping factor } \\
\text { (Point 40) }\end{array}$ & $\begin{array}{c}\text { Damping factor } \\
\text { difference }(\%)\end{array}$ \\
\hline 1 & 64.63889 & 64.58773 & 0.05116 & 0.001152 & 0.001335 & 15.87176 \\
\hline 2 & 392.3132 & 393.5316 & 1.2184 & 0.00645 & 0.005472 & 15.16743 \\
\hline 3 & 459.1516 & 459.0252 & 0.1264 & 0.000667 & 0.000651 & 2.477419 \\
\hline 4 & 1015.351 & - & - & 0.00753 & - & - \\
\hline 5 & 1125.492 & 1126.48 & 0.988 & 0.00459 & 0.007716 & 68.11871 \\
\hline 6 & 1147.168 & 1148.179 & 1.011 & 0.003201 & 0.00217 & 32.22889 \\
\hline 7 & 1252.44 & - & - & 0.005597 & - & - \\
\hline 8 & 1419.851 & 1419.687 & 0.164 & 0.000415 & 0.000545 & 31.51598 \\
\hline 9 & 2175.132 & - & - & 0.002125 & - & - \\
\hline 10 & 2524.38 & 2523.689 & 0.691 & 0.000578 & 0.000676 & 16.9878 \\
\hline
\end{tabular}

FRF signals and the differences between the modal parameters. The modal frequency difference is the absolute difference between the modal frequencies of the two denoised FRF signals. The modal damping factor difference is calculated by equation (21). The extracted modal frequencies of the two signals are nearly the same, and the max difference between the extracted modal frequencies of the two denoised FRF signals is $1.21 \mathrm{~Hz}$, which shows the validity of extracted modal frequencies. For the damping factors, the max difference between them is the difference at mode 5 , which is $68 \%$. The main reason is thought that the mode 5 of Point 40 loses a large part of its amplitude in the noise elimination, and the correlated damping factor is estimated to be higher than the real one. Then, for the other damping factors, the differences between them are not big, and the max difference is $32 \%$, which shows that the extracted damping factors are also validated. Then, the analysis shows that the curve fitting of the two denoised FRF signals is precise, and the extracted modal parameters match each other, which further proves the validity of the noise reduction.

\section{Conclusion}

In this paper, a novel noise reduction method based on the modal peak-based Hankel-SVD technique (MPHSVD) is proposed, which aims to acquire the precise FRF signal from the noised FRF signal for the modal identification. The proposed method contains one accumulation iteration to select the component signals which cover the whole modal modes and some residue noise and one reselection iteration to separate the component signals of the modal modes from the residue noise for completing the noise reduction. The advantage of the method is that the component signals correlated with the modal modes can be determined by the two iterations. The simulated FRF of a plate with the added heavy noise is taken as an example to show how the denoising method works. The noise removal rate reaches above $93 \%$, and the further modal analysis shows that the modal frequencies and modal damping factors are extracted precisely. Besides, the detailed analysis of the correlation between the component signals and the modal modes and effect of different bandwidths for the noise reduction are also investigated. The simulation analysis of FRFs contaminated with various degrees of Gaussian noise shows that the noise reductions are fully successful for FRFs with SNRs of $7.68 \mathrm{~dB}$ and $3.25 \mathrm{~dB}$, respectively. The application of two experimental FRF signals of a flat plate in the high-temperature environment also shows the superiority and effectiveness of the proposed method. However, some amplitudes of the modal modes contaminated with too much noise decrease after the noise elimination. Nevertheless, the method shows the practical potential to engineering application in the modal test.

\section{Data Availability}

Data are available on request.

\section{Conflicts of Interest}

The authors declare that there are no conflicts of interest regarding the publication of this paper.

\section{Acknowledgments}

The authors gratefully appreciate the financial support for this work provided by the National Natural Science Foundation of China and the National Safety Academic Foundation of China (no. U1730129). The supports from the Jiangsu Province Key Laboratory of Aerospace Power System and the Key Laboratory of Aero-Engine Thermal Environment and Structure, Ministry of Industry and Information Technology, are also gratefully acknowledged.

\section{References}

[1] D. He, X. Wang, M. I. Friswell, and J. Lin, "Identification of modal parameters from noisy transient response signals," Structural Control and Health Monitoring, vol. 24, no. 11, pp. 1-21, 2017.

[2] X. Bao and C. Shi, "Ambient vibration responses denoising for operational modal analysis of a jacket-type offshore platform," Ocean Engineering, vol. 172, pp. 9-21, 2019.

[3] H. Pehlivan, "Frequency analysis of gps data for structural health monitoring observations," Structural Engineering \& Mechanics, vol. 66, no. 2, pp. 185-193, 2018.

[4] L. I. Wei, J. Xiaolin, C. Haibo et al., "Denosing method of mine microseismic signal based on EEMD_Hankel_SVD," Journal of China Coal Society, vol. 43, no. 7, pp. 1910-1917, 2018. 
[5] A. Cavallini, G. Suganya, S. J. Subbhaiya, K. Kannan, and J. Jaikanth, "Development of Hankel-SVD hybrid technique for multiple noise removal from PD signature," IET Science, Measurement and Technology, vol. 13, no. 8, pp. 1075-1084, 2019.

[6] A. Nicolson and K. K. Paliwal, "Deep learning for minimum mean-square error approaches to speech enhancement," Speech Communication, vol. 111, pp. 44-55, 2019.

[7] T. G. Yadava and H. S. Jayanna, "Speech enhancement by combining spectral subtraction and minimum mean square error-spectrum power estimator based on zero crossing," International Journal of Speech Technology, vol. 22, no. 3, pp. 639-648, 2018.

[8] R. Ali and M. Moonen, "A contingency multi-microphone noise reduction strategy based on linearly constrained multichannel wiener filtering," in Paper Presented at the 2016 International Workshop on Acoustic Signal Enhancement (IWAENC), Xi'an, China, September 2016.

[9] Y. Yang and C. Bao, "RS-CAE-based AR-Wiener filtering and harmonic recovery for speech enhancement," IEEE/ACM Transactions on Audio, Speech, and Language Processing, vol. 27, no. 11, pp. 1752-1762, 2019.

[10] Y. Pan, L. Zhang, X. Wu, K. Zhang, and M. J. Skibniewski, "Structural health monitoring and assessment using wavelet packet energy spectrum," Safety Science, vol. 120, pp. 652-665, 2019.

[11] H.-T. Fang and D.-S. Huang, "Noise reduction in lidar signal based on discrete wavelet transform," Optics Communications, vol. 233, no. 1-3, pp. 67-76, 2004.

[12] N. Qiao, L.-h. Wang, Q.-y. Liu et al., "Multi-scale eigenvalues Empirical Mode Decomposition for geomagnetic signal filtering," Measurement, vol. 146, pp. 885-891, 2019.

[13] H. Rong, Y. Gao, L. Guan, Q. Zhang, F. Zhang, and N. Li, "GAM-based mooring alignment for SINS based on an improved CEEMD denoising method," Sensors (Basel), vol. 19, no. 16, 2019.

[14] F. A. Castaño, A. M. Hernández, and G. Soto-Romero, "Assessment of artifacts reduction and denoising techniques in electrocardiographic signals using ensemble average-based method," Computer Methods and Programs in Biomedicine, vol. 182, Article ID 105034, 2019.

[15] R. Kashid and K. P. Paradeshi, "Design of effective algorithm for EMG artifact removal from multichannel EEG data using ICA and wavelet method," Advances in Intelligent Systems and Computing, vol. 941, pp. 955-964, 2020.

[16] T. Pham, Y. S. Lee, S. Mathulaprangsan, and J. C. Wang, "Source separation using dictionary learning and deep recurrent neural network with locality preserving constraint," in Paper Presented at the Proceedings of the IEEE International Conference on Multimedia and Expo, pp. 151-156, Hong Kong, China, August 2017.

[17] W. Yuan, B. He, S. Wang, J. Wang, and M. Unoki, "Enhanced feature network for monaural singing voice separation," Speech Communication, vol. 106, pp. 1-6, 2019.

[18] M. Garcia-Fernandez, Y. Alvarez-Lopez, A. Arboleya-Arboleya et al., "SVD-based clutter removal technique for GPR," in Paper Presented at the 2017 IEEE Antennas and Propagation Society International Symposium, July 2017.

[19] S. Vanlanduit, B. Cauberghe, P. Guillaume, P. Verboven, and E. Parloo, "Reduction of large frequency response function data sets using a robust singular value decomposition," Computers \& Structures, vol. 84, no. 12, pp. 808-822, 2006.
[20] K. Y. Sanliturk and O. Cakar, "Noise elimination from measured frequency response functions," Mechanical Systems and Signal Processing, vol. 19, no. 3, pp. 615-631, 2005.

[21] X. Zhao and B. Ye, "Similarity of signal processing effect between Hankel matrix-based SVD and wavelet transform and its mechanism analysis," Mechanical Systems and Signal Processing, vol. 23, no. 4, pp. 1062-1075, 2009.

[22] W.-X. Yang and P. W. Tse, "Development of an advanced noise reduction method for vibration analysis based on singular value decomposition," NDT \& E International, vol. 36, no. 6, pp. 419-432, 2003.

[23] D. Fan, C. Lv, and Q. Cai, "SVD filter based on noise singular values clustering," in Paper Presented at the 2010 International Conference on Intelligent Computation Technology and Automation, pp. 665-668, Changsha, China, May 2010.

[24] X. Zhao and B. Ye, "Selection of effective singular values using difference spectrum and its application to fault diagnosis of headstock," Mechanical Systems and Signal Processing, vol. 25, no. 5, pp. 1617-1631, 2011.

[25] X. X. Bao, C. L. Li, and C. B. Xiong, "Noise elimination algorithm for modal analysis," Applied Physics Letters, vol. 107, no. 4, Article ID 041901, 2015.

[26] Z. Qiao and Z. Pan, "SVD principle analysis and fault diagnosis for bearings based on the correlation coefficient," Measurement Science and Technology, vol. 26, no. 8, Article ID 085014, 2015.

[27] M. Zhao and X. Jia, "A novel strategy for signal denoising using reweighted SVD and its applications to weak fault feature enhancement of rotating machinery," Mechanical Systems and Signal Processing, vol. 94, pp. 129-147, 2017.

[28] X. Zhao and B. Ye, "Separation of single frequency component using singular value decomposition," Circuits, Systems, and Signal Processing, vol. 38, no. 1, pp. 191-217, 2018.

[29] L. Cheng, T. Liang, L. Guo, M. Cheng, and L. Zeng, "Resonance enhanced singular value decomposition and its application to the vibration monitoring of turboprop engine," Zhendong Yu Chongji/Journal of Vibration and Shock, vol. 37, no. 22, pp. 206-213, 2018.

[30] D. Formenti and M. Richardson, "Parameter estimation from frequency response measurements using rational fraction polynomials (twenty years of progress)," in Proceedings of SPIE - the International Society for Optical Engineering, Orlando, FL, USA, November 1982.

[31] P. Martin and S. Rothberg, "Introducing speckle noise maps for laser vibrometry," Optics and Lasers in Engineering, vol. 47, no. 3-4, pp. 431-442, 2009. 